\title{
Design and optimization of a novel bent-vane type radio frequency quadrupole
}

\author{
Lei Yang $\odot,{ }^{1,2}$ Liang Lu, ${ }^{1,2,3,{ }^{*}}$ Zhouli Zhang, ${ }^{1, \dagger}$ Chaochao Xing, ${ }^{1,2}$ Yulu Huang, ${ }^{1}$ Tao He, ${ }^{1,2}$ \\ Chenxing Li, ${ }^{1}$ Xianbo Xu, ${ }^{1}$ Liepeng Sun, ${ }^{1}$ Longbo Shi, ${ }^{1}$ Andong $\mathrm{Wu},{ }^{1}$ and $\mathrm{Yuan} \mathrm{He}^{1}$ \\ ${ }^{1}$ Institute of Modern Physics, Chinese Academy of Sciences, Lanzhou 730000, China \\ ${ }^{2}$ School of Nuclear Science and Technology, University of Chinese Academy of Sciences, \\ Beijing 100049, China \\ ${ }^{3}$ Sino-French Institute of Nuclear Engineering and Technology, Sun Yat-sen University, \\ Zhuhai 519000, China
}

(Received 18 June 2019; accepted 16 January 2020; published 10 February 2020)

\begin{abstract}
A novel radio frequency quadrupole (RFQ) resonant structure with bent vanes is proposed at the Institute of Modern Physics, Chinese Academy of Sciences (IMP, CAS). Compared with the traditional four-vane type RFQs, the bent-vane type structure is more compact at low frequency with significant reduction in the cross section size, and is capable of using a simple cooling system due to its rotationally symmetric structure. An $81.25 \mathrm{MHz}$ proof-of-principle cavity is now under development at IMP. The beam dynamics design, electromagnetic optimizations with multipacting simulations, multiphysics analyses, and mechanical design of this novel bent-vane type RFQ (BV-RFQ) will be presented in this paper.
\end{abstract}

DOI: 10.1103/PhysRevAccelBeams.23.021301

\section{INTRODUCTION}

Radio frequency quadrupole (RFQ) is widely used as the injector for high energy accelerator due to its outstanding capabilities in strong focusing, bunching, and accelerating simultaneously for low velocity beams with high quality output.

Mainly, there are two types of RFQ structures, the fourvane type and the four-rod type. The four-vane type RFQ structures are mainly used in high frequency cases with reasonable section sizes and simple but efficient cooling systems, which are usually operated at high duty factor and continuous wave $(\mathrm{CW})$ modes. The four-rod type RFQ structures are usually employed in the low frequency cases with acceptable section sizes, and are mostly used in the low duty factor operations, which are limited by the complicated cooling systems [1-3].

However, RFQs with low frequencies but running at $\mathrm{CW}$ modes are required by some projects, such as the $80.5 \mathrm{MHz}$ RFQ for the Facility for Rare Isotope Beams (FRIB) at the Michigan State University (MSU) [4] and the $81.25 \mathrm{MHz}$

\footnotetext{
Corresponding author. luliang3@mail.sysu.edu.cn

Corresponding author. zhangzhouli@impcas.ac.cn
}

Published by the American Physical Society under the terms of the Creative Commons Attribution 4.0 International license. Further distribution of this work must maintain attribution to the author(s) and the published article's title, journal citation, and DOI.
RFQ for the Low Energy Accelerator Facility (LEAF) at IMP, CAS [5-7]. Four-vane structures were adopted for both RFQs to get effective coolings for CW operations, but with quite large section diameters, which is $766.9 \mathrm{~mm}$ for the FRIB RFQ, and is $721 \mathrm{~mm}$ for the LEAF RFQ. To reduce the transverse dimensions of the four-vane type RFQs in low frequencies and to separate the operating frequencies from the nearby modes, Argonne National Laboratory (ANL) proposed a hybrid RFQ structure which equipped the conventional four-vane structure with coupling windows in vanes and split-coaxial cavities. The transverse diameter was reduced to $500 \mathrm{~mm}$ for a 57.5 MHz RFQ. But the cooling system is complicated in this kind of structure [8].

To get a small section size at low frequency and a simple cooling system at the same time, a novel RFQ structure with bent vanes has been proposed at IMP. The conceptual section view of the new RFQ structure is shown in Fig. 1.

According to the equivalent circuit of the four-vane RFQ structure in cloverleaf geometry, as shown in Eq. (1),

$$
r^{2}=\frac{16}{\mu_{0}(4+3 \pi) \omega_{0}^{2} C^{\prime}}
$$

where $r$ is the section radius, $\omega_{0}$ is the resonant frequency, $C^{\prime}$ is the total capacitance per unit length and $\mu_{0}$ is the permeability constant. The section size of the RFQ could be reduced by increasing the cavity capacitance when the frequency is fixed [9]. For the BV-RFQ structure, additional capacitances could be generated among the bent vanes, which is expected to reduce the section size. 


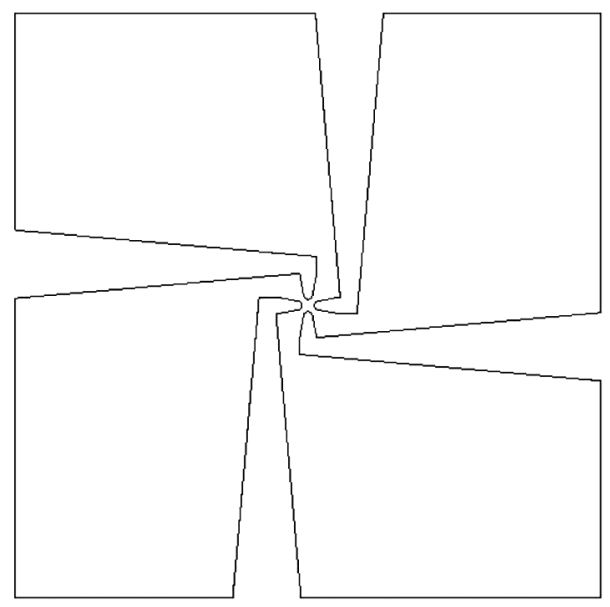

FIG. 1. The conceptual section view of the BV-RFQ.

And similar to the four-vane structure, the BV-RFQ is rotationally symmetric, thus a simple cooling system could be efficient for CW operation.

An $81.25 \mathrm{MHz}$ proof-of-principle cavity is built to demonstrate this new concept. With this prototype cavity, the reduction of the cavity section size could be verified with exact quantitative analyses, the beam dynamics and rf performance with bent-vane structure could be validated, the efficiency of a simple cooling system could be confirmed.

In this paper, the design and optimization of the 81.25 MHz BV-RFQ prototype cavity will be discussed in detail. The beam dynamics design and the electromagnetic optimizations with multipacting analyses will be presented in Secs. II and III. The multi-physics analyses will be described in Sec. IV. The mechanical design will be summarized in Sec. V. And finally, the summary and outlook will be given in Sec. VI.

\section{BEAM DYNAMICS DESIGN}

\section{A. Beam dynamics parameters}

The beam dynamics design of the $81.25 \mathrm{MHz}$ BV-RFQ prototype cavity was performed by RFQGen [10]. This prototype cavity is proposed to accelerate $5 \mathrm{~mA} \mathrm{He}{ }^{+}$ $(q / A=1 / 4)$ beam from $4 \mathrm{keV} / \mathrm{u}$ to $42.5 \mathrm{keV} / \mathrm{u}$, main beam parameters are listed in Table I. The intervane voltage is selected to be $70 \mathrm{kV}$ due to the rf source limitation and thermal management considerations, which seems to be reasonable for $\mathrm{CW}$ operation. The maximum surface peak electric field is $18.23 \mathrm{MV} / \mathrm{m}$, which is 1.73 times higher than the Kilpatrick limit $\left(E_{k}=10.529 \mathrm{MV} / \mathrm{m}\right.$ @ $81.25 \mathrm{MHz}$ ). This value is not modest but still reasonable to avoid the risk of voltage breakdown. The transverse emittance growth is $10 \%$ in x-plane and is 5\% in y-plane, which is really small for the acceptance of the following devices.
TABLE I. The dynamics parameters of the prototype cavity.

\begin{tabular}{lc}
\hline \hline Parameter & Value \\
\hline Particle & $\mathrm{He}^{+}(q / A=1 / 4)$ \\
Frequency [MHz] & 81.25 \\
Beam current [mA] & 5 \\
Input energy [keV/u] & 4 \\
Output energy [keV/u] & 42.5 \\
Vane voltage [kV] & 70 \\
Vane length [mm] & 530.07 \\
Kilpatrick factor & 1.73 \\
Minimum aperture [mm] & 3.444 \\
Average aperture [mm] & 5.23 \\
Synchronous phase & $-90^{\circ}-30^{\circ}$ \\
Modulation factor & $1-2.07$ \\
$\epsilon_{t}$ (norm.RMS. entrance) [cm mrad] & 0.02 \\
$\epsilon_{t x}$ (norm.RMS. exit) [cm mrad] & 0.022 \\
$\epsilon_{t y}($ norm.RMS. exit) [cm mrad] & 0.021 \\
$\epsilon_{l}$ (norm.RMS. exit) $[\mathrm{MeV} \mathrm{deg]}$ & 0.0816 \\
Transmission efficiency [\%] & 96.9 \\
\hline \hline
\end{tabular}

The evolution of main dynamics parameters to cell number is shown in Fig. 2, where $m$ is the modulation, $a$ is the minimum radial aperture, $B$ is the focusing parameter, $\phi_{s}$ is the synchronous phase and $W_{s}$ is the energy of the synchronous particle. It is preferring to fixing the transverse focusing strength (B) thus to make the tuning procedure easier, and also to decrease the manufacture difficulty with the possibility of machining the vane modulations with a simple 2D cutter.

Multiparticle dynamics simulations were done with RFQGen with 30000 macroparticles. The simulation results of beam transmission are shown in Fig. 3. The transmission efficiency is $96.9 \%$ at $5 \mathrm{~mA}$.

Figure 4 shows the phase-space projections at the entrance and exit of the RFQ. It is clearly that the exit particle distribution is concentrated, the energy spread is within $\pm 0.01 \mathrm{MeV}$ and the phase spread is within $\pm 30^{\circ}$.

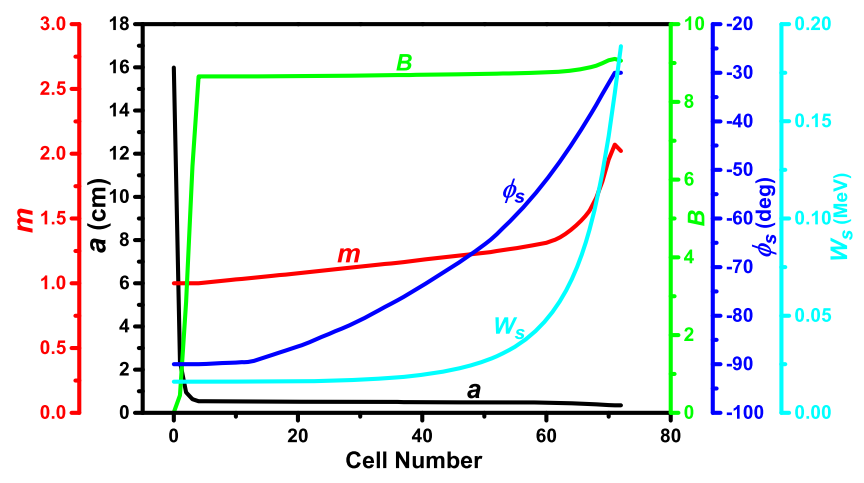

FIG. 2. The main dynamics parameters along the prototype cavity. 
BENT-VANE TYPE RFQ, 81.25MHz, He+, 5mA, 70kV -- Lei Yang, RFQ group, 2018
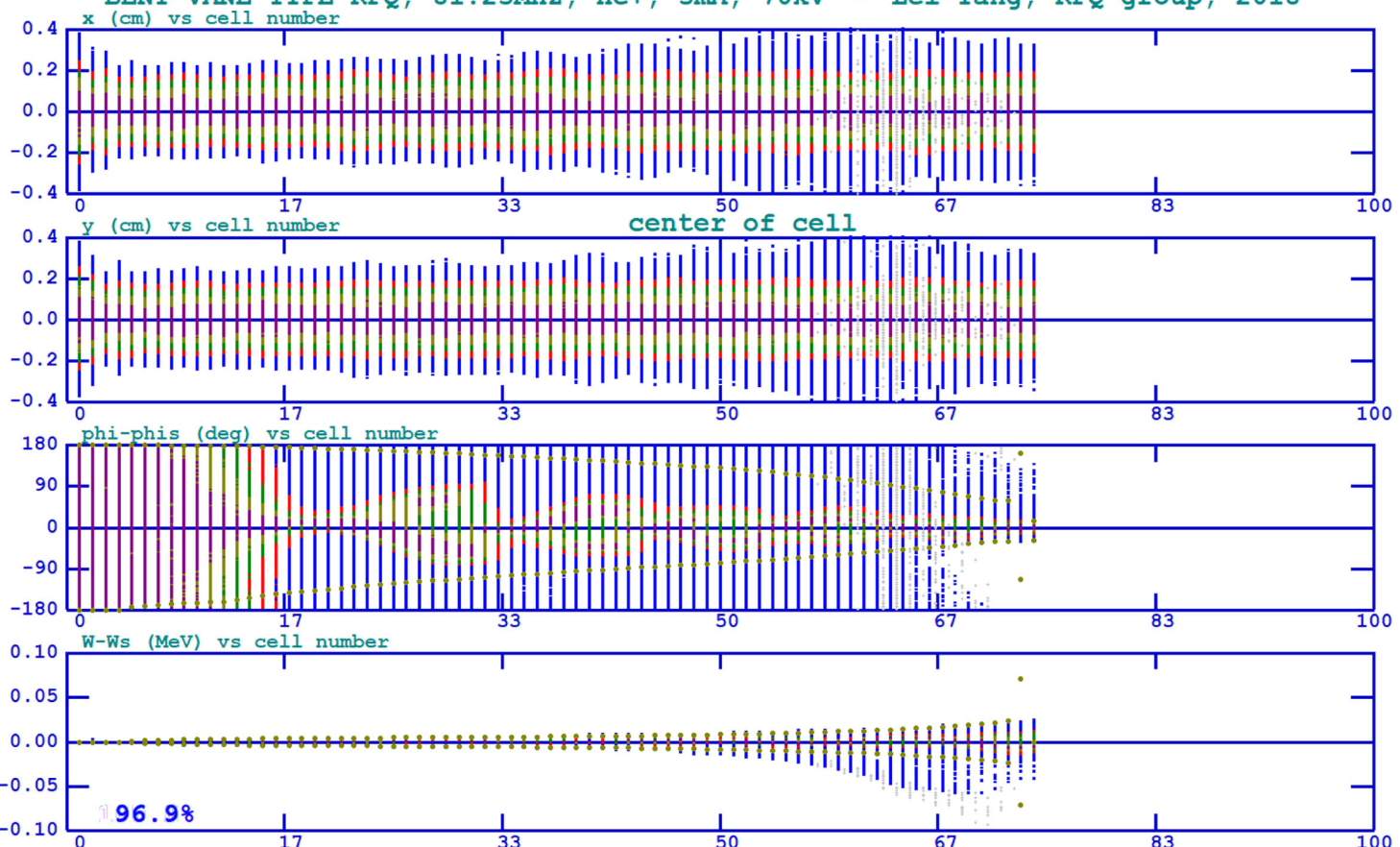

FIG. 3. Beam dynamics simulation results of the prototype cavity (RFQGen). Plots from top to bottom are the $\mathrm{x}$ plane beam envelope, the y plane beam envelope, the phase and the energy spread versus cell number, respectively.

BENT-VANE TYPE RFQ, 81.25MHz, He+, 5mA, 70kV -- Lei Yang, RFQ group, 2018
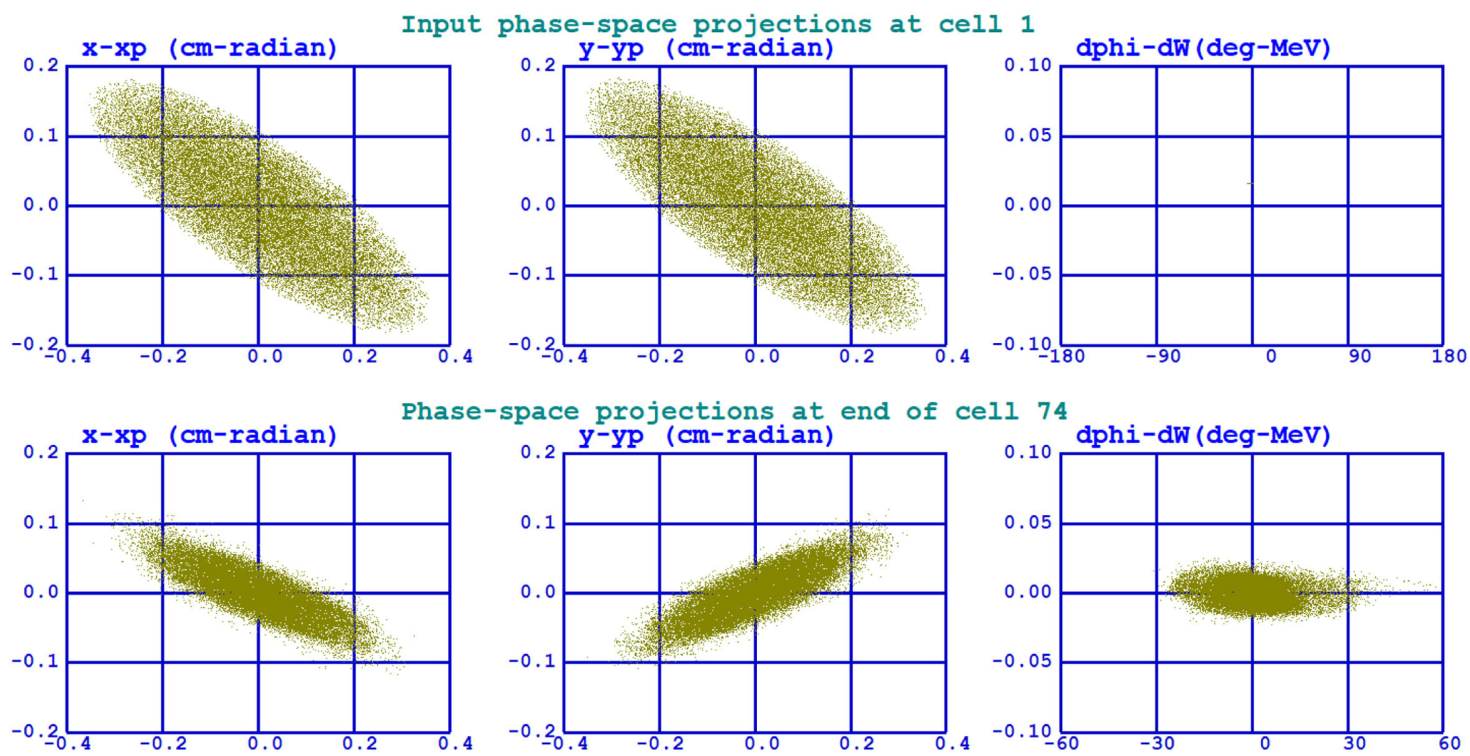

FIG. 4. The phase-space projections at entrance (top) and exit (bottom) of the prototype cavity.

\section{B. Tolerance analysis}

Above discussions about the dynamics design are based on the ideal situation. Figure 5 shows the tolerance analyses for this BV-RFQ prototype cavity. Figure 5(a) shows the transmission efficiency for different beam currents. The transmission efficiency stays at a high level above 95\% when the beam current varies from 0 to $8 \mathrm{~mA}$. From $8 \mathrm{~mA}$ to $15 \mathrm{~mA}$, the transmission efficiency decreases dramatically due to the space charge effect but is still larger than $90 \%$. Increasing the beam current to $30 \mathrm{~mA}$, the transmission efficiency drops to $72 \%$ rapidly. Figure 5(b) presents the transmission efficiency for different energy spread. The transmission efficiency remains almost unchanged at around $96.8 \%$ within $11 \%$ energy spread 


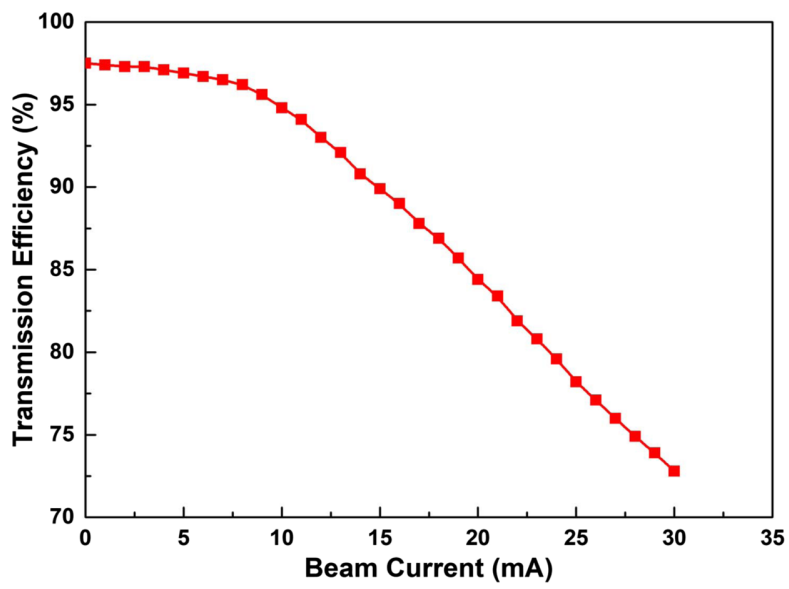

(a) Beam transmission efficiency versus beam current.

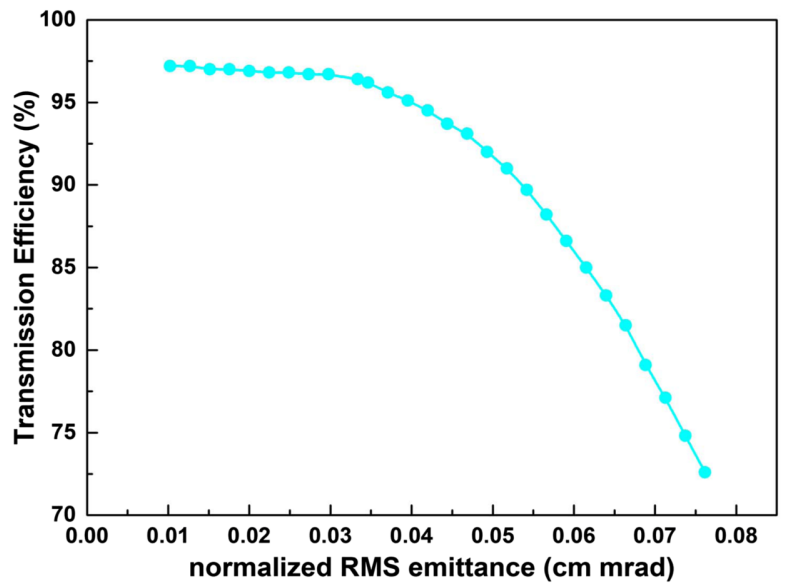

(c) Beam transmission efficiency versus normalized RMS emittance.

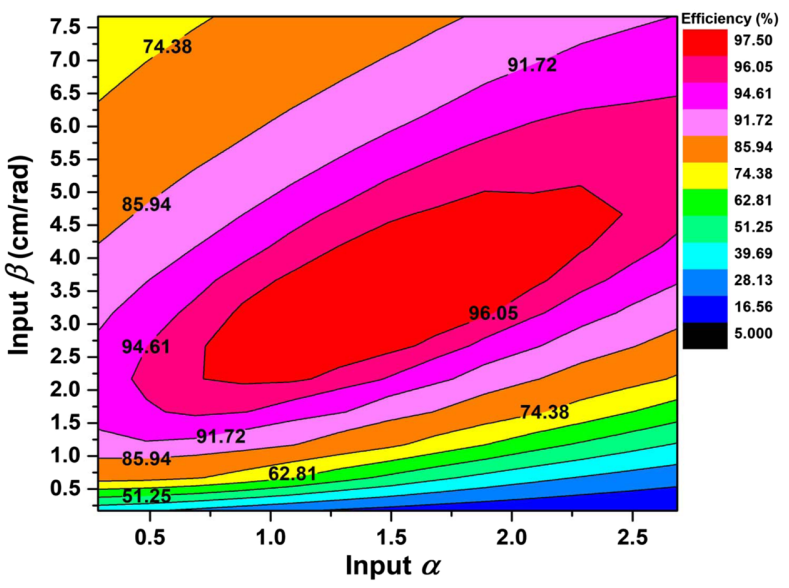

(e) Beam transmission efficiency versus twiss parameters.

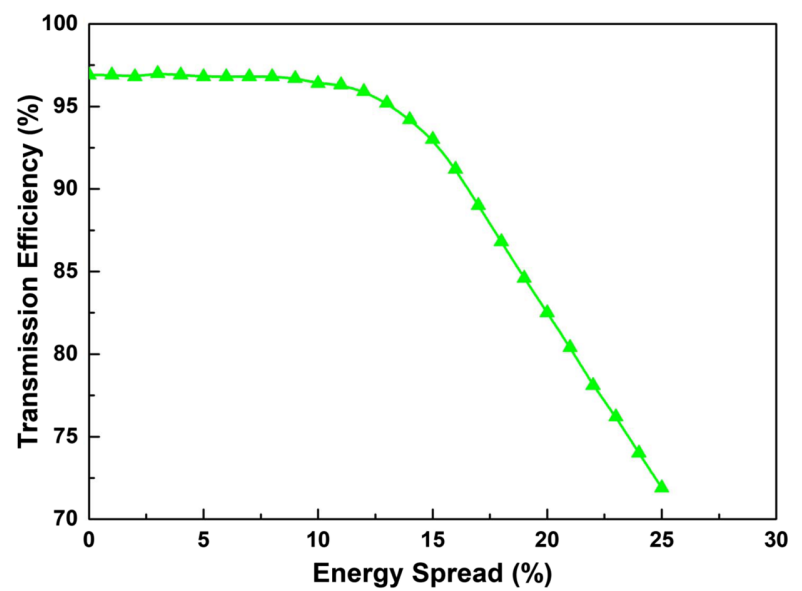

(b) Beam transmission efficiency versus energy spread.

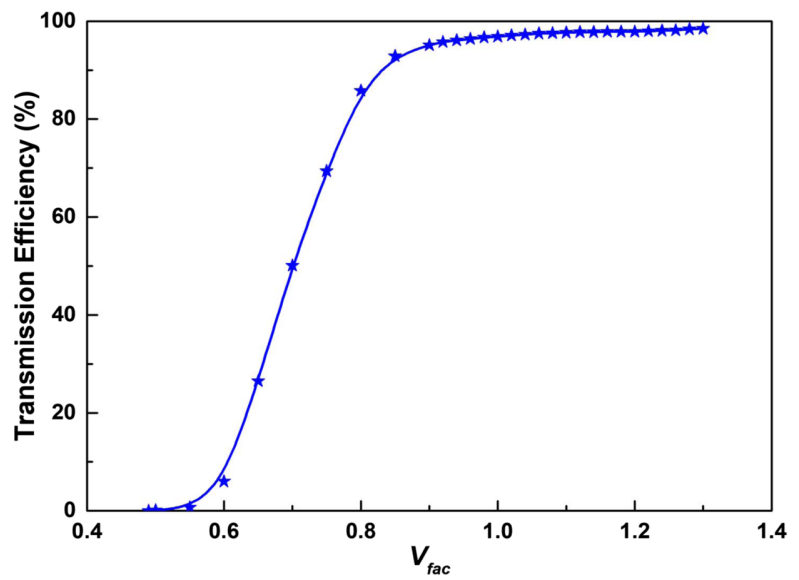

(d) Beam transmission efficiency versus vane voltage.

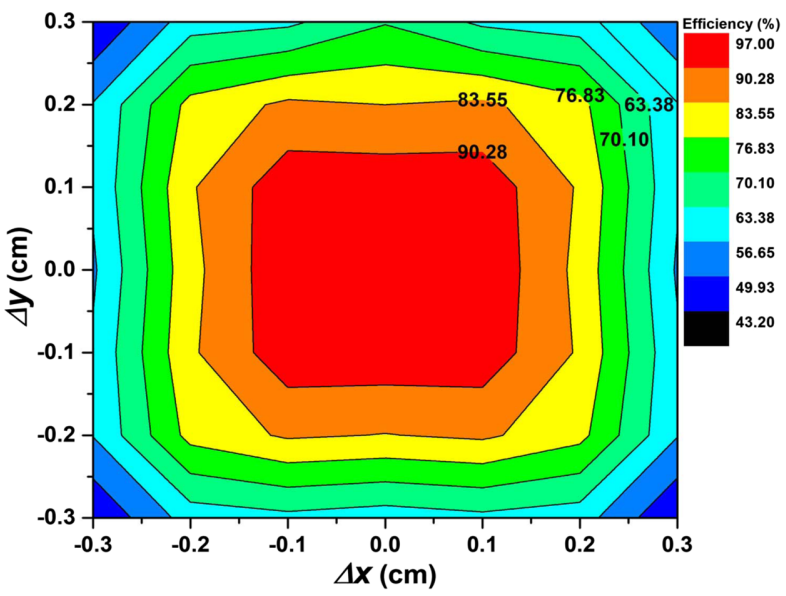

(f) Beam transmission efficiency versus spatial displacements.

FIG. 5. Tolerance analyses for the dynamics design of the prototype cavity.

and reduces to about $71 \%$ with $25 \%$ energy spread. Figure 5(c) describes the transmission efficiency for different normalized RMS emittance of the input beam. The transmission efficiency is $96.9 \%$ for the matched input RMS emittance of $0.02 \mathrm{~cm}$ mrad, the efficiency is almost the same with the RMS emittance varying from 0.01 to $0.035 \mathrm{~cm} \mathrm{mrad}$, and is down to $72.6 \%$ if the emittance is increased to $0.076 \mathrm{~cm}$ mrad. Figure $5(\mathrm{~d})$ gives the beam transmission efficiency for different vane voltages $\left(V_{\text {fac }}\right.$ is the normalization factor of the simulation voltage to the design voltage). The beam transmission efficiency ascends along with the increasing vane voltage, but it is preferring to keeping the voltage fluctuation within $\pm 1 \%$ of the design value due to rf source limitation and thermal 


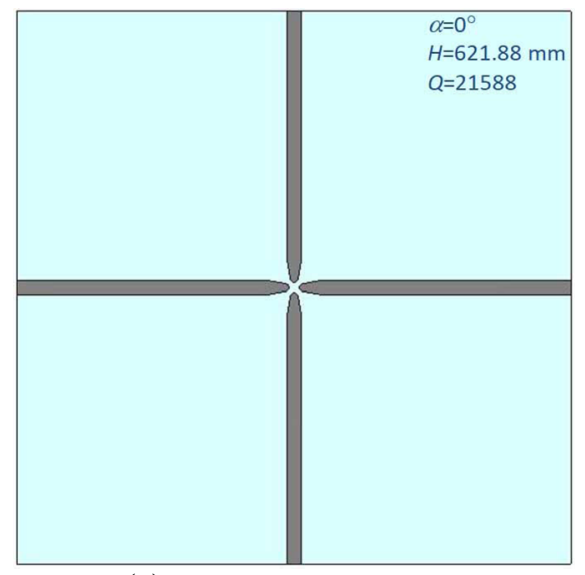

(a) $\alpha=0^{\circ}$ (four-vane type)

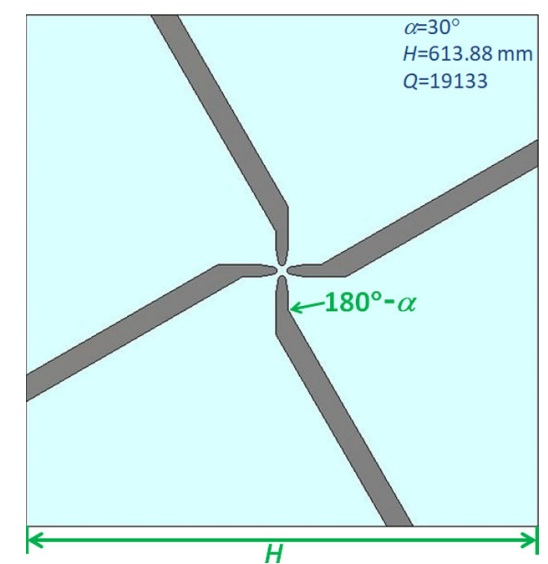

(b) $\alpha=30^{\circ}$ (bent-vane type)

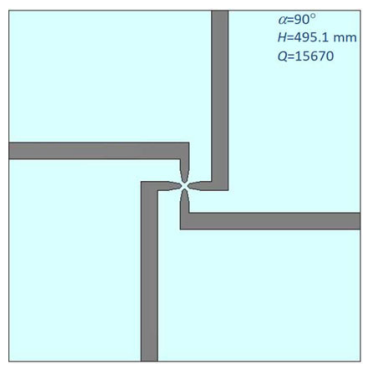

(c) $\alpha=90^{\circ}$ (bent-vane type)

FIG. 6. The conceptual demonstration of the BV-RFQ cross sections for different bent angles.

considerations. Figure 5(e) shows the beam transmission efficiency for different input twiss parameters $(\alpha, \beta)$ at the RFQ entrance, the transmission efficiency is $96.9 \%$ at the design point $(1.2849,3.1677 \mathrm{~cm} / \mathrm{rad})$ and is more than $96.05 \%$ in a large range. Figure 5(f) shows the effect of spatial displacements on the transmission efficiency. It is necessary to keep the spatial displacements within $\pm 1 \mathrm{~mm}$ to make sure the transmission efficiency is more than $90 \%$.

From the tolerance analyses, the dynamics design keeps great performance in a large margin.

\section{RF-EM DESIGN}

The electromagnetic (EM) studies of the BV-RFQ prototype cavity were completed with CST MWS [11]. High quality factor and high shunt impedance were pursued during the optimization thus less rf power in need and lower heat load need to be removed. Frequency correcting and cross section profile optimization were done with a thin $(1 \mathrm{~mm}$ ) full slice model (not $1 / 4$ due to the nonaxisymmetric cross section of the structure). The tuner period

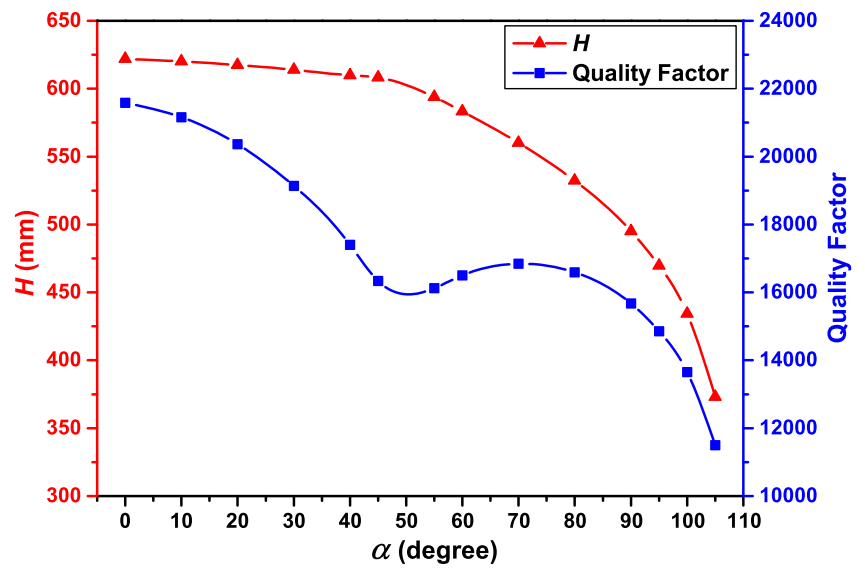

FIG. 7. The variation of the cross section width $(H)$ and quality factor along the change of bent angle $(\alpha)$. optimization and the field flatness tuning were carried out in a full length model with contributions of every component.

\section{A. Conceptual demonstration}

Firstly, considering the overall cross section profile of the prototype cavity. A square cross section with planar surfaces is preferring in this design due to its benefits for mechanical processing and assembling.

Compared with the traditional four-vane type RFQs, the vanes of the BV-RFQ are bent thus resulting to a compact

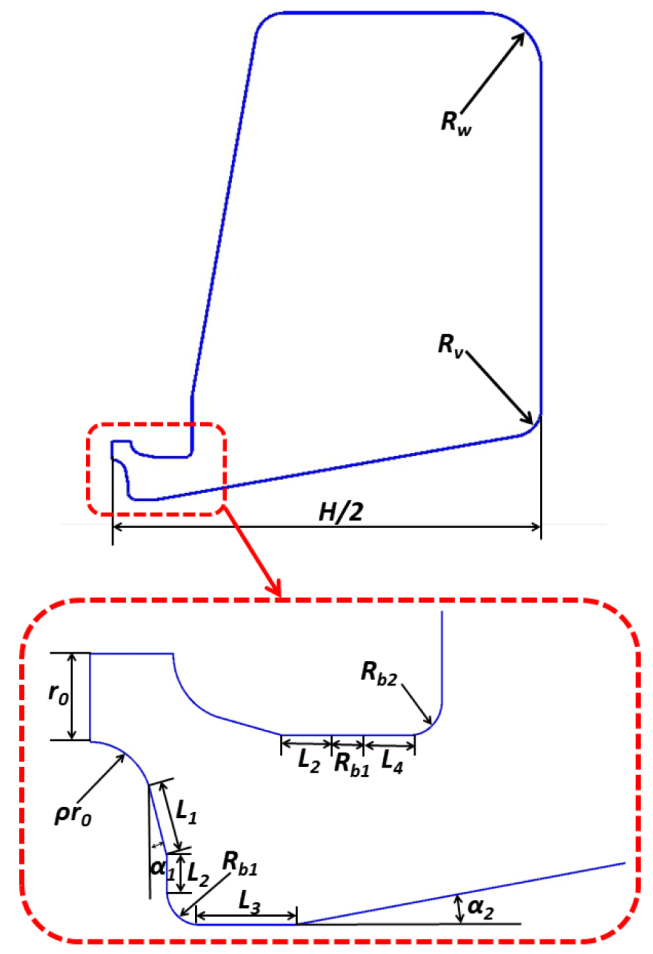

FIG. 8. The cross section profile for a single vane of the BV-RFQ. 


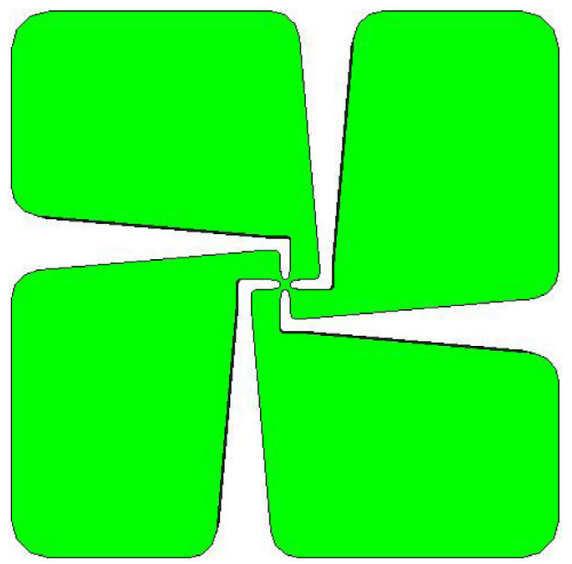

FIG. 9. Full slice model for the BV-RFQ prototype cavity optimization.

size in low frequency. Figures 6 and 7 shows the cross section width $H$ for different bent angles $\alpha$ with frequency fixed to $81.25 \mathrm{MHz}$ for each case. As the bent angle increases, the cross section width decreases gradually. It also could be seen that the quality factor decreases simultaneously with slight fluctuation around 50 degrees. Increasing the bent angle from 0 degree to 105 degrees, $40 \%$ reduction on the cross section width could be achieved along with $47 \%$ quality factor dropping. A final angle of 90 is chosen as a balance of cross section size and quality

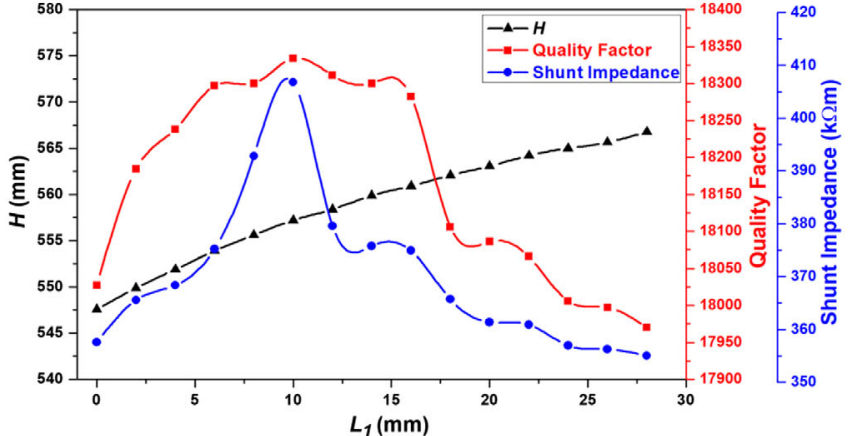

(a) Parameter: $L_{1}$

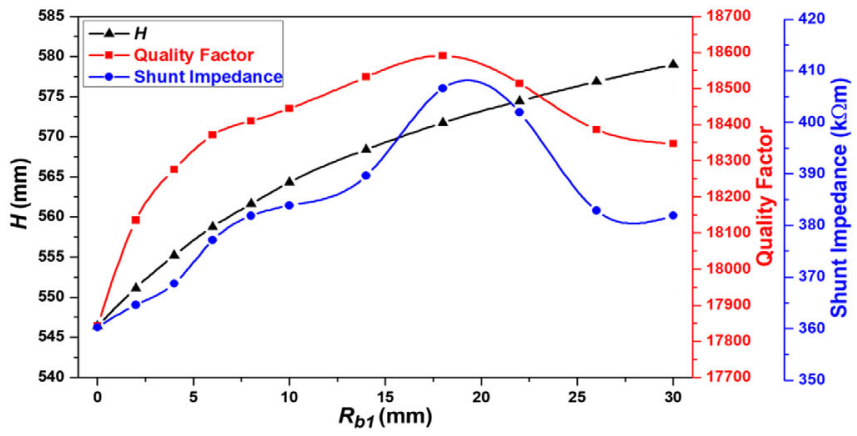

(c) Parameter: $R_{b 1}$ factor, which is also an appropriate value for machining. At this angle, the cross section width is turned out to be reduced by $20 \%$, the quality factor is decreased by $27 \%$.

\section{B. Thin slice model optimization}

The full geometry of the cross section for a single vane could be defined by 13 independent parameters, as shown in Fig. 8 [12]. Here $r_{0}, \rho$ and $\alpha_{1}$ were defined in RFQGen, other ten left parameters were optimized in CST MWS. Unlike the traditional four-vane type RFQs, the BV-RFQ is the nonaxisymmetric cross section thus a full slice model $(1 \mathrm{~mm})$ is built for the parametric optimization, as shown in Fig. 9.

During the simulation, the frequency was fixed to 81.25 MHz. Figure 10 (a, b, c and d) shows the variation of the section size $H$, quality factor and shunt impedance with the vane geometry parameters $L_{1}, L_{2}, R_{b 1}$ and $\alpha_{2}$. With the increasing of $L_{1}, L_{2}$ and $R_{b 1}$, the section width $H$ increases monotonically while the quality factor and shunt impedance have peak values around $10 \mathrm{~mm}, 17 \mathrm{~mm}$ and $17 \mathrm{~mm}$, respectively. The section width $H$ increases with the increasing of $\alpha_{2}$ while the quality factor and shunt impedance decreases simultaneously. The optimizations of other parameters are similar. Final optimized geometry parameters are summarized in Table II, which are determined based on the balance of rf performance, cavity size and machining processing difficulty.

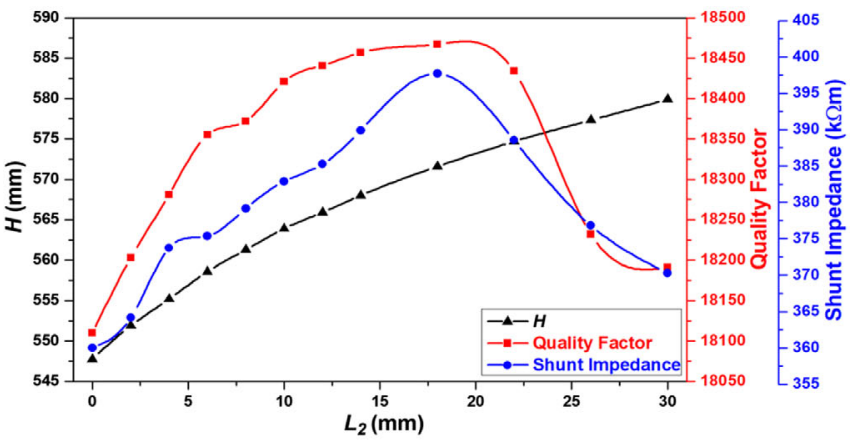

(b) Parameter: $L_{2}$

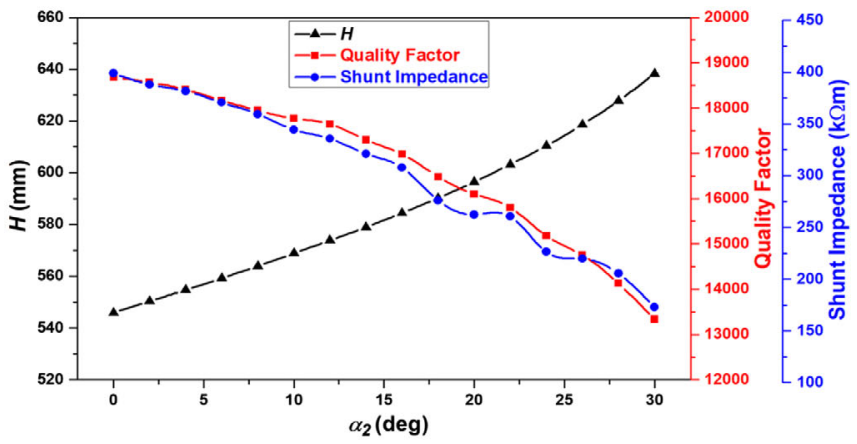

(d) Parameter: $\alpha_{2}$

FIG. 10. The variation of the $H$, quality factor and shunt impedance with vane geometry parameters of a slice model. (a) vane geometry parameter $L_{1}$; (b) vane geometry parameter $L_{2}$; (c) vane geometry parameter $R_{b 1}$; (d) vane geometry parameter $\alpha_{2}$. (Only four parameters are presented here). 
TABLE II. The optimized vane geometry parameters of BV-RFQ prototype cavity cross section.

\begin{tabular}{lccc}
\hline \hline Parameter & Value & Parameter & Value \\
\hline$r_{0}$ & $5.347 \mathrm{~mm}$ & $H$ & $567.34 \mathrm{~mm}$ \\
$\rho$ & 0.75 & $R_{v}$ & $30 \mathrm{~mm}$ \\
$\alpha_{1}$ & $10 \mathrm{deg}$ & $\alpha_{2}$ & $5 \mathrm{deg}$ \\
$L_{1}$ & $10 \mathrm{~mm}$ & $R_{w}$ & $40 \mathrm{~mm}$ \\
$L_{2}$ & $12 \mathrm{~mm}$ & $L_{4}$ & $10 \mathrm{~mm}$ \\
$R_{b 1}$ & $5 \mathrm{~mm}$ & $R_{b 2}$ & $5 \mathrm{~mm}$ \\
$L_{3}$ & $10 \mathrm{~mm}$ & & \\
\hline \hline
\end{tabular}

The BV-RFQ is working at $T E_{210}$-like mode as the traditional four-vane type RFQ, as shown in Fig. 11, thus particles could be accelerated, focused, and bunched at the same time. With the unnormalized electric field directly exported from CST MWS, the voltages between adjacent electrodes could be calculated, which are nearly the same. It indicates that the BV-RFQ has fine electric field quality near the electrode tips.

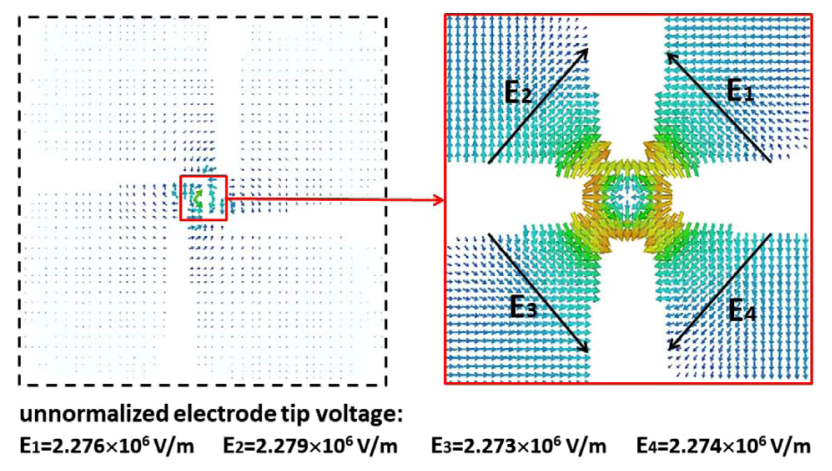

FIG. 11. The electric field distribution near electrode tips.

\section{Full length model optimization}

With the optimized vane geometry parameters from the slice model, a full length model could be obtained, as shown in Fig. 12. Here the undercut dimensions were modified to get a fine field flatness.

It is crucial to make sure the cavity resonate at the target frequency during the operation, plunger tuners are proposed in this BV-RFQ to compensate the frequency shift due to the manufacturing errors and other perturbations during the installation. Two plunger tuners are employed in each quadrant thus resulted to a total tuner number of eight for this whole prototype cavity. The diameter of the plunger is $90 \mathrm{~mm}$, the distance separation of the adjacent plungers is $265.035 \mathrm{~mm}$ in one quadrant.

The tuning range and the quality factor are very sensitive to the separation distance between the tuner and the vane base (callout $d$ in Fig. 12). The closer of the tuner to the vane base, a larger tuning range could be achieved, while a bigger dropping of the quality factor and a greater probability of multipacting at the same insertion depth. Figure 13 shows the frequency shift and quality factor decreasing versus the insertion depth of the plunger tuners with different separation distance $d$. It is also obvious to see that the tuning range and the quality factor change a little from $173 \mathrm{~mm}$ to $238 \mathrm{~mm}$ of the separation distance $d$. A final separation of $173 \mathrm{~mm}$ is chosen based on the above considerations, with a tuning sensitivity of $12.67 \mathrm{kHz} / \mathrm{mm}$. The tuners are inserted $25 \mathrm{~mm}$ into the magnetic field area with the selected separation distance, as shown in Fig. 14.

The field flatness is very sensitive to the undercut dimensions. With careful optimizations, the final undercut dimensions are shown in Fig. 15. The electric field is flatted with $H c u t=83 \mathrm{~mm}, \alpha_{3}=65$ degrees, $D_{\text {in }}=$ $120 \mathrm{~mm}$ and $D_{\text {out }}=120 \mathrm{~mm}$. Figure 16 presents the
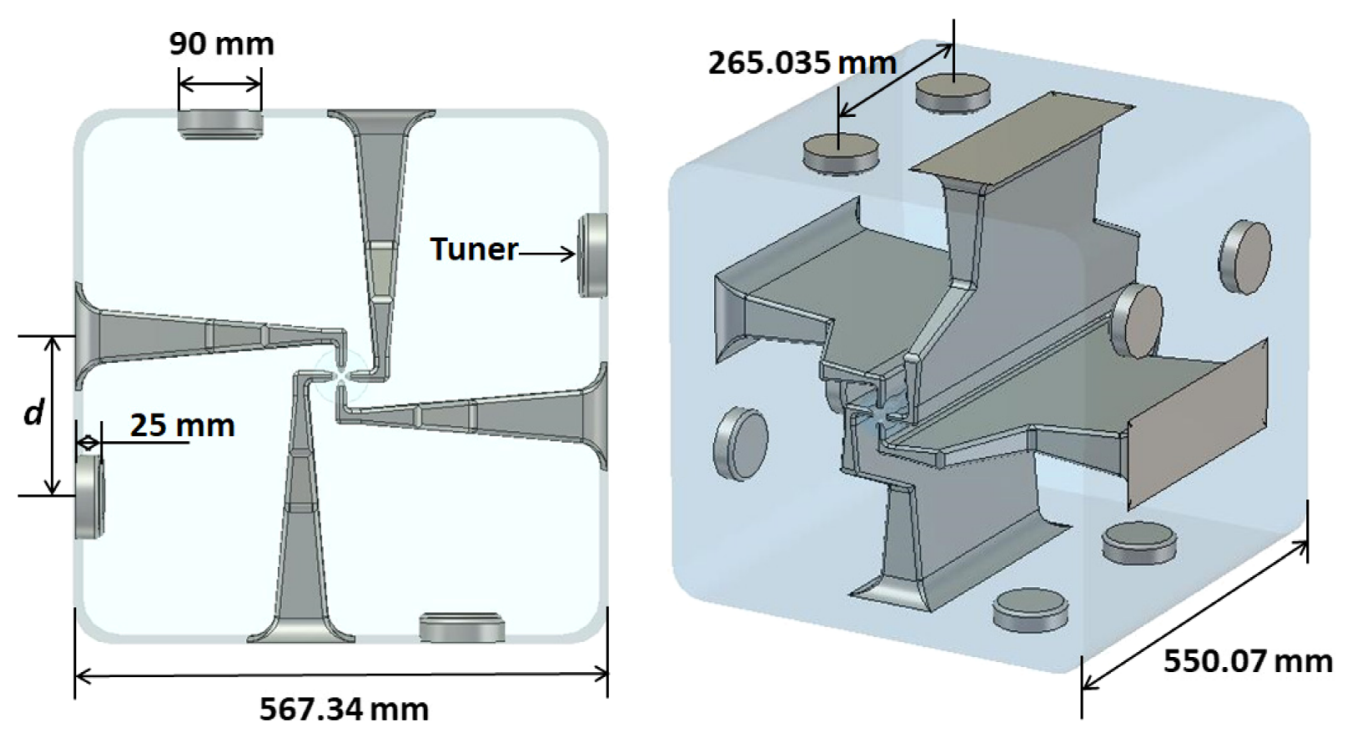

FIG. 12. Full length model of the BV-RFQ prototype cavity. 


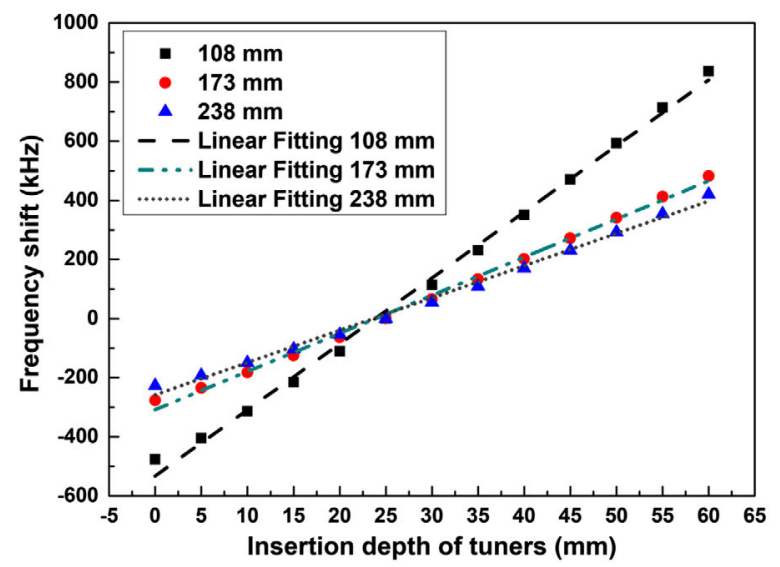

(a)

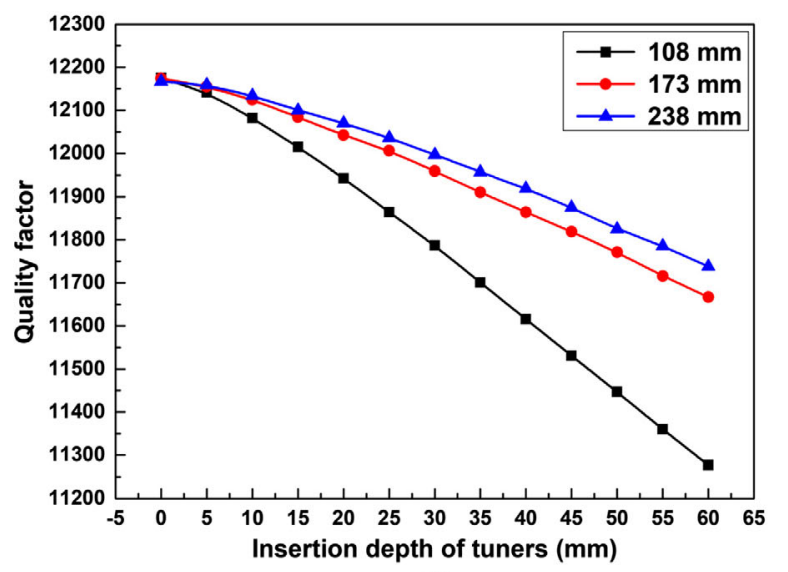

(b)

FIG. 13. The tuning range of all tuners (a) and the quality factor of the cavity (b) for different insertion depth of tuners and different separation distance between tuner and vane base.

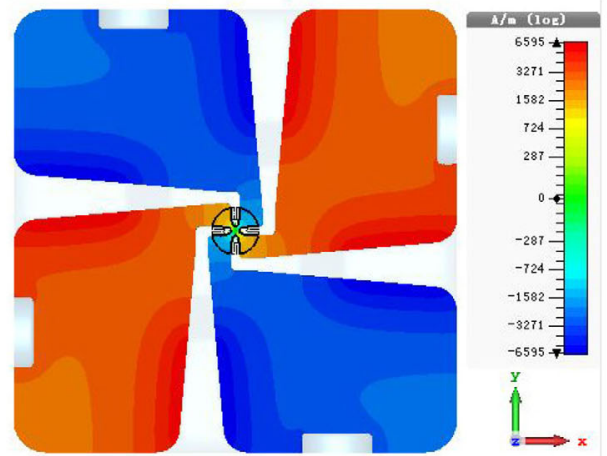

Quadrupole mode: magnetic field

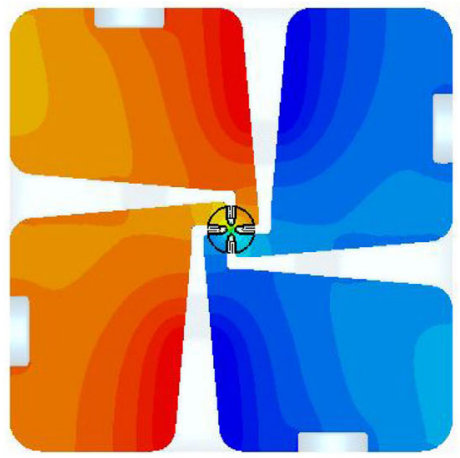

Dipole mode: magnetic field

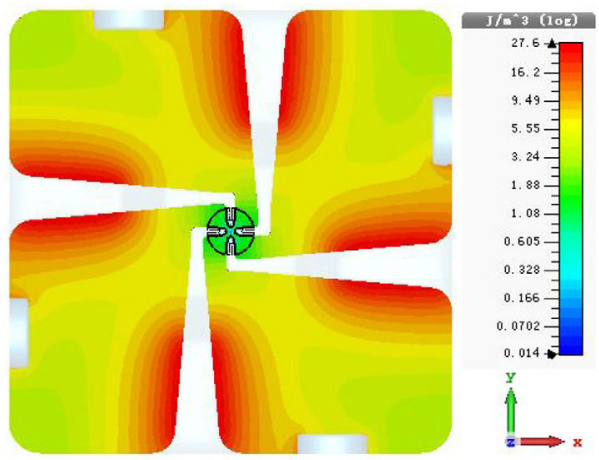

Quadrupole mode: magnetic field energy density

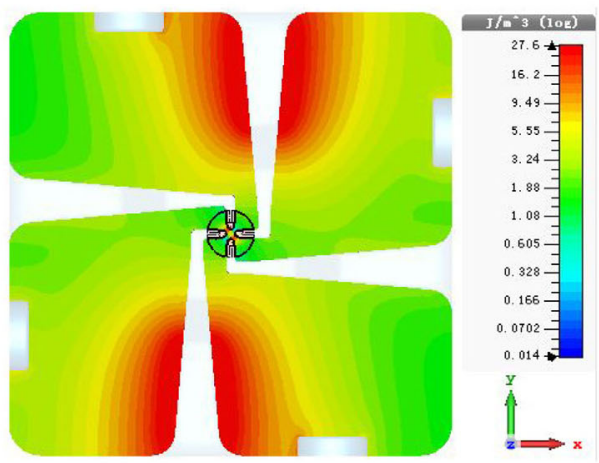

Dipole mode: magnetic field energy density

FIG. 14. The magnetic field distribution for the quadrupole mode and the dipole mode in the BV-RFQ prototype cavity.

normalized electric field distribution (off-axis $10 \mathrm{~mm}$ between adjacent vanes) along the beam direction with and without undercuts.

The electromagnetic parameters of the full length prototype cavity model with vane modulations are listed in Table III. The nearest dipole mode is $91.858 \mathrm{MHz}$, which is $10.609 \mathrm{MHz}$ higher than the operation mode. Mode separation schemes like vane coupling rings, $\pi$-mode stabilizing loops and dipole stabilization rods could be omitted in this BV-RFQ prototype cavity. The Kilpatrick factor is 1.69 in the CST simulation, which is almost the same to the value 1.73 in beam dynamics design. The shunt impedance of this BV-RFQ is $269.8 \mathrm{k} \Omega \mathrm{m}$, which results to a total power loss of $9990 \mathrm{~W}$ at the design voltage $70 \mathrm{kV}$. Figure 17 presents the designed shunt impedance of the major RFQs worldwide [2,5,13-21], the performance of this BV-RFQ is between the four-rod type RFQ and the four-vane type RFQ. 


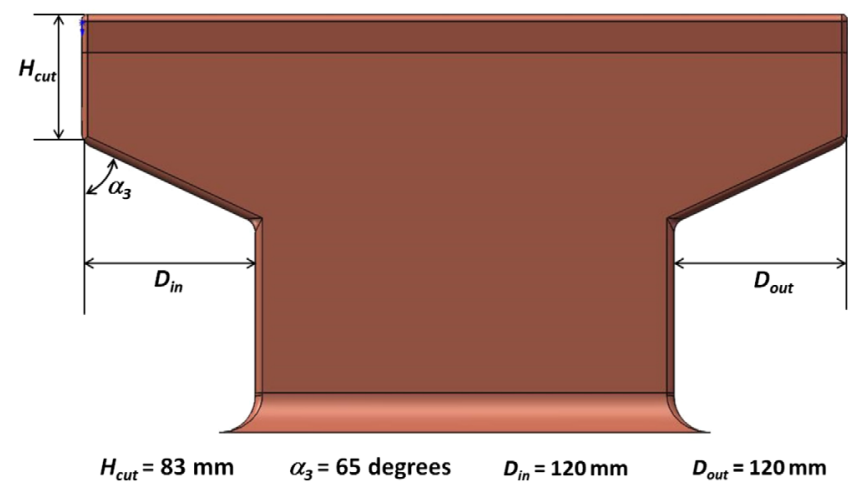

FIG. 15. The undercut schematic diagram of the BV-RFQ prototype cavity.

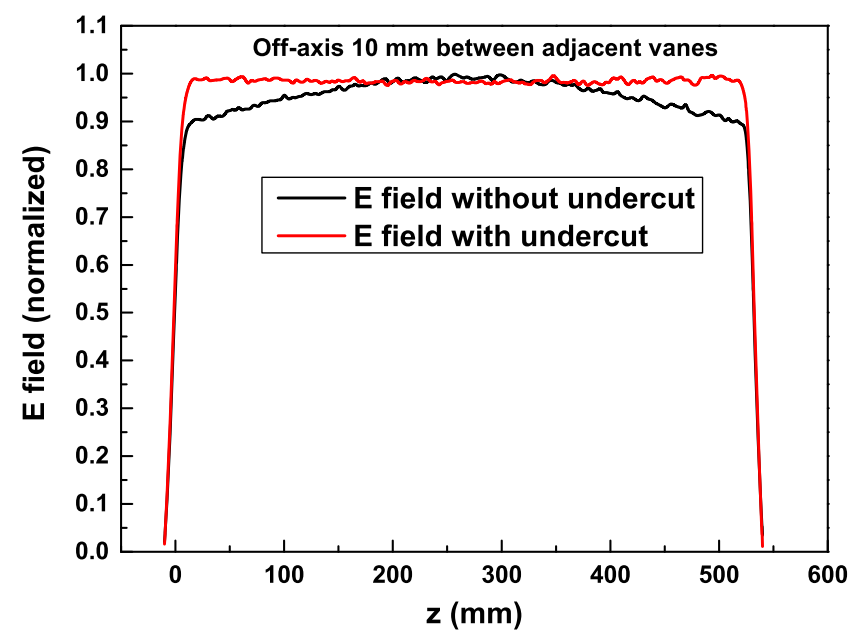

FIG. 16. The normalized electric field distribution along the beam direction with and without undercuts.

\section{Beam dynamics verification}

With the electromagnetic fields exported from CST Eigenmode solver, beam dynamics verification was done with TraceWin [22]. During the simulation, a rectangular domain $(6 \mathrm{~mm} \times 6 \mathrm{~mm} \times 550.07 \mathrm{~mm})$ near the electrode tips was selected in the full length model with refined

TABLE III. rf electromagnetic parameters of the full length BV-RFQ prototype cavity.

\begin{tabular}{lc}
\hline \hline Parameter & Value \\
\hline Frequency [MHz] & 81.249 \\
Frequency without inserting tuners [MHz] & 80.972 \\
Nearest dipole mode frequency [MHz] & 91.858 \\
Quality factor & 12003 \\
Shunt impedance $[\mathrm{k} \Omega \mathrm{m}]$ & 269.8 \\
Power loss [W] & 9990 \\
Peak electric field $[\mathrm{MV} / \mathrm{m}]$ & 17.805 \\
Kilpatrick factor $(\mathrm{CST}$ simulation) & 1.69 \\
$H$ [mm] & 567.34 \\
\hline \hline
\end{tabular}

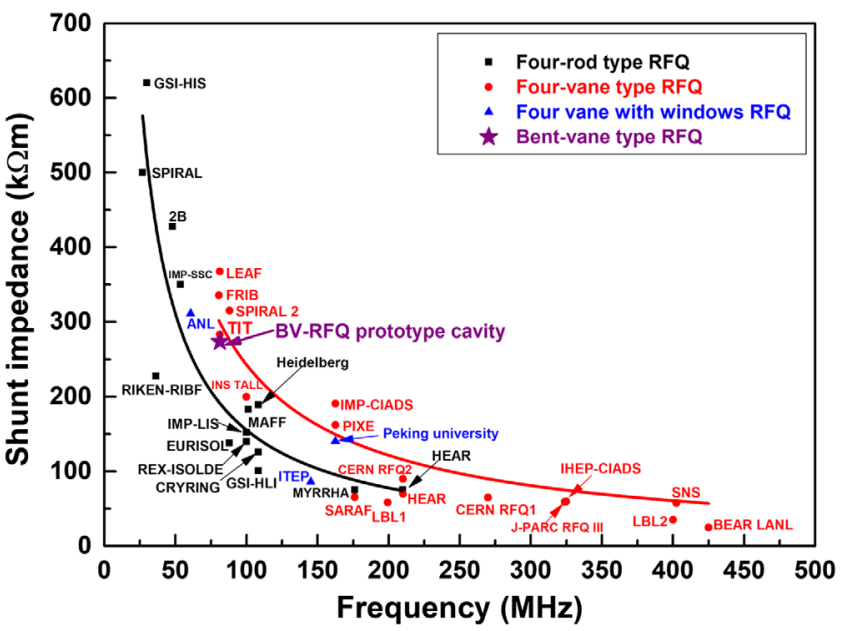

FIG. 17. The designed shunt impedance of main RFQs in the world.

meshes. The electromagnetic fields were calculated and exported with $25 \times 25 \times 1000$ sampling points. The input beam parameters were gotten from RFQGen, which are listed in Table IV.

Tracking results are shown in Fig. 18, the normalized transverse and longitudinal emittance at the exit of the RFQ are summarized in Table $\mathrm{V}$, with comparisons to the parameters from RFQGen. The transverse and longitudinal emittance are nearly the same from both codes. The transmission efficiency is $0.3 \%$ smaller in TraceWin. But in general, the tracking results from both codes maintain great consistency.

\section{E. Multipacting analyses}

Multipacting is a common phenomenon in rf structures that secondary electrons emitting and increasing exponentially when primary electrons hitting on the rf surface. Abundant electrons absorbing a large quantity of energy, thus the required electromagnetic field could not be established. Most times, multipacting could be conditioned during the rf conditioning procedure, it is still necessary to investigate the possibility of multipacting happening during the EM design stage.

Multipacting analyses of this BV-RFQ prototype cavity were done with the Furman-Pivi model [23] in CST Particle Studio [11]. During the simulation, electromagnetic field maps of the prototype cavity were calculated in the Eigenmode solver with refined mesh at the rf surface.

TABLE IV. The input beam parameters in TraceWin.

\begin{tabular}{ll}
\hline \hline Parameter & Value \\
\hline$\epsilon_{t}($ norm.RMS. entrance) [cm mrad] & 0.02 \\
$\alpha_{x}$ and $\alpha_{y}$ & 1.2849 \\
$\beta_{x}$ and $\beta_{y}[\mathrm{~cm} / \mathrm{rad}]$ & 3.1677 \\
\hline \hline
\end{tabular}



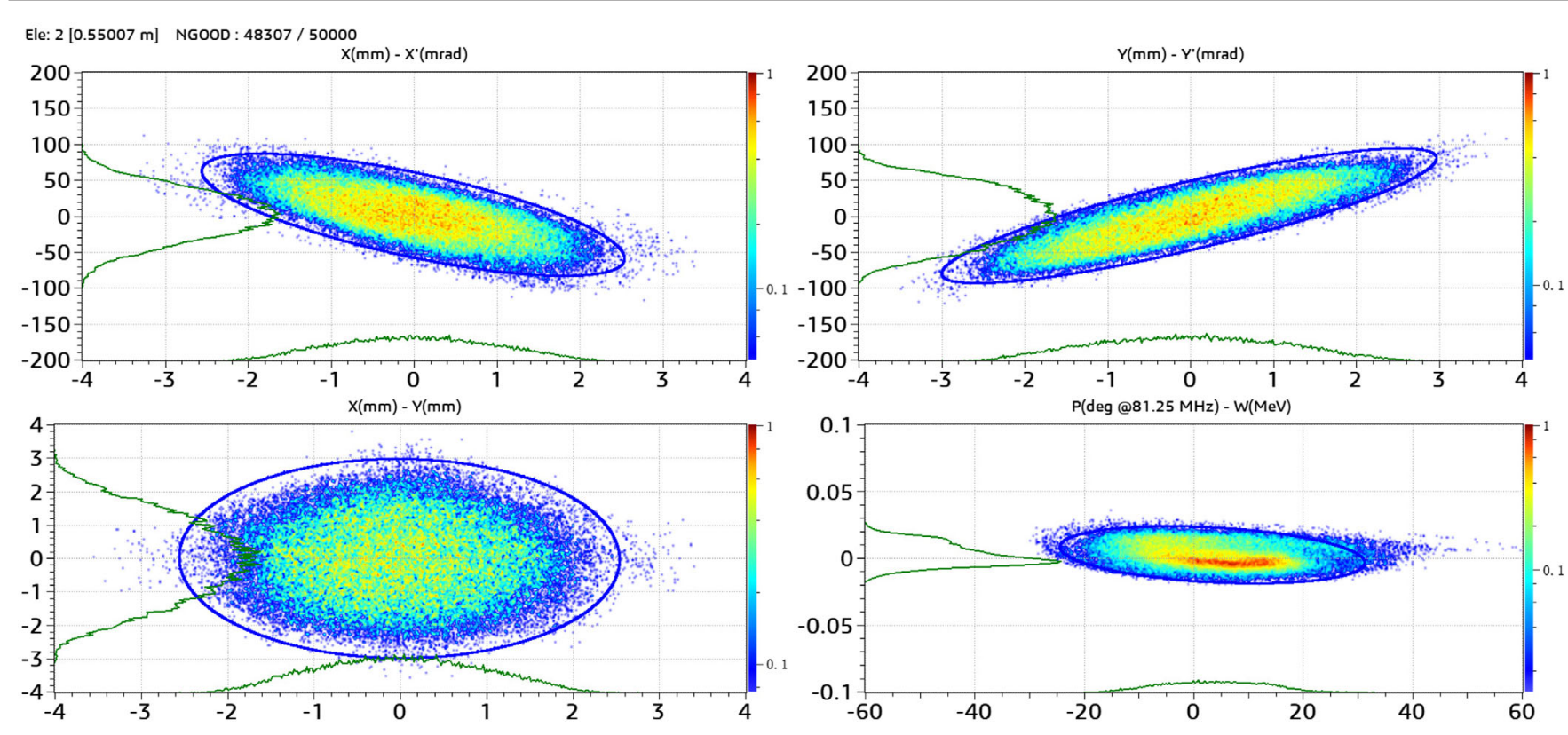

FIG. 18. The output beam distributions in TraceWin.

These field maps were then being scaled to the design level and being exported to the particle studio. Particle source was defined at the suspicious surface area, as shown in Fig. 19.

The primary electrons are Gaussian distribution and the emission energies are ranging from $2.5 \mathrm{eV}$ to $7.5 \mathrm{eV}$ [24]. The averaged secondary emission yield $\langle\mathrm{SEY}\rangle$ factors at different power levels could then be calculated to evaluate the occurrence of multipacting, which is defined as:

$$
\langle\mathrm{SEY}\rangle=\frac{I_{\text {emission }}}{I_{\text {collision }}}
$$

The particle traces of secondary electrons and the $\langle\mathrm{SEY}\rangle$ factors could be calculated either in Particle Tracking (PT) solver or in Particle-In-Cell (PIC) solver. PIC solver has perfect post processing but takes more time to complete the calculation. In order to reduce simulation time and improve computational efficiency, a $150 \mathrm{~mm}$ long model was used for multipacting simulations.

The results of multipacting simulations are shown in Fig. 20. The power factor is defined as the normalization factor of the simulation power relative to the designed

TABLE V. The output beam parameters in TraceWin and RFQGen.

\begin{tabular}{lcc}
\hline \hline Parameter & $\begin{array}{c}\text { Value } \\
\text { (TraceWin) }\end{array}$ & $\begin{array}{c}\text { Value } \\
\text { (RFQGen) }\end{array}$ \\
\hline$\epsilon_{t x}$ (norm.RMS. exit) [cm mrad] & 0.02217 & 0.022 \\
$\epsilon_{t y}$ (norm.RMS. exit) [cm mrad] & 0.0211 & 0.021 \\
$\epsilon_{l}$ (norm.RMS. exit) [MeV deg] & 0.0818 & 0.0816 \\
Transmission efficiency [\%] & 96.6 & 96.9 \\
\hline \hline
\end{tabular}

power. It can be obtained that the $\langle\mathrm{SEY}\rangle$ values are greater than 1 for almost all power points except at $1 \%$ designed power level. It seems that multipacting exists at almost all power levels in this prototype cavity. It is crucial for us to pay special attention during the rf conditioning procedure. Further optimizations on the rf structure to reduce the occurrence of multipacting are on the way.

\section{MULTIPHYSICS ANALYSES}

From the rf parameters listed in Table III, the total power loss is $9990 \mathrm{~W}$. Consider the quality factor dropping during the manufacture, installing and operation, this power loss could be much higher. The power is transmitted to the heat load on the rf surface finally, this heat needs to be taken away by water or other cooling material, thus the cavity could be operated at a stable environment. Multiphysics analyses with the water-cooling channel design will be discussed in this section.

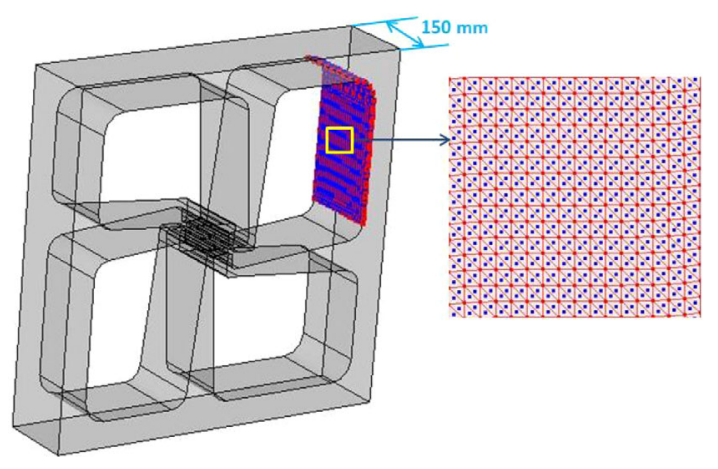

FIG. 19. Particle source area. 


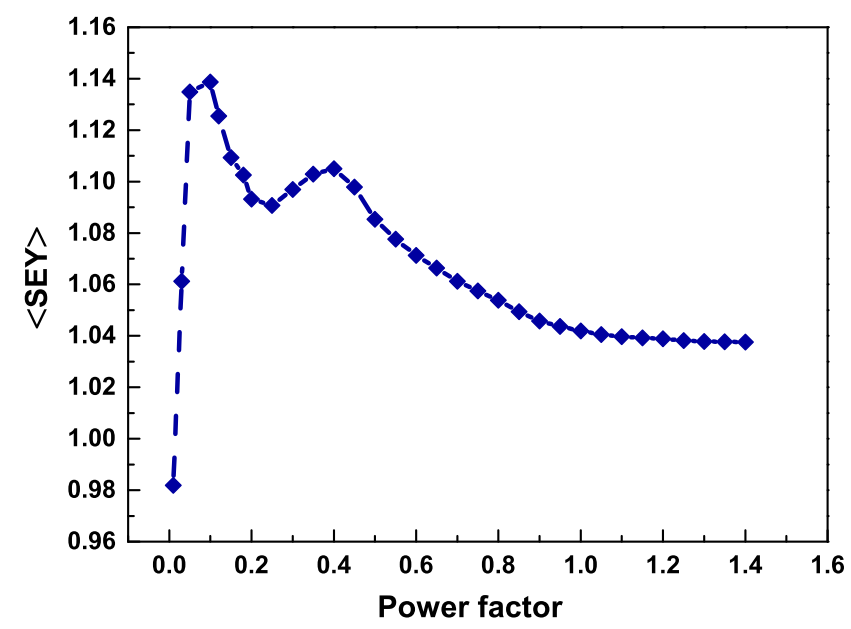

FIG. 20. The results of multipacting simulation in the BV-RFQ prototype cavity.

The multiphysics analyses of this BV-RFQ were done with ANSYS Workbench [25]. To make a better understanding of the relationship among electromagnetic field, heating, deformation, stress, and frequency shifting, a coupled analyses scheme is established which includes the electromagnetic analysis, thermal analysis, and structure analysis, as shown in Fig. 21. During the simulation, the surface power losses calculated in High Frequency Structure Simulator (HFSS) were exported to the SteadyState Thermal solver and scaled to the operation level. The temperature distribution at the rf surface was calculated and transmitted to the static structural solver, the deformation information was gotten and finally be fed back to HFSS, the frequency shift could then be calculated.

The goal of the multiphysics analyses is to design the water cooling channel. From the electromagnetic analyses results, power losses on different parts of the cavity are summarized in Table VI. Compared with the power losses on the vanes and walls, the power loss on the tuners is almost negligible and cooling on the tuners could be omitted. Four vane cooling channels and twelve wall

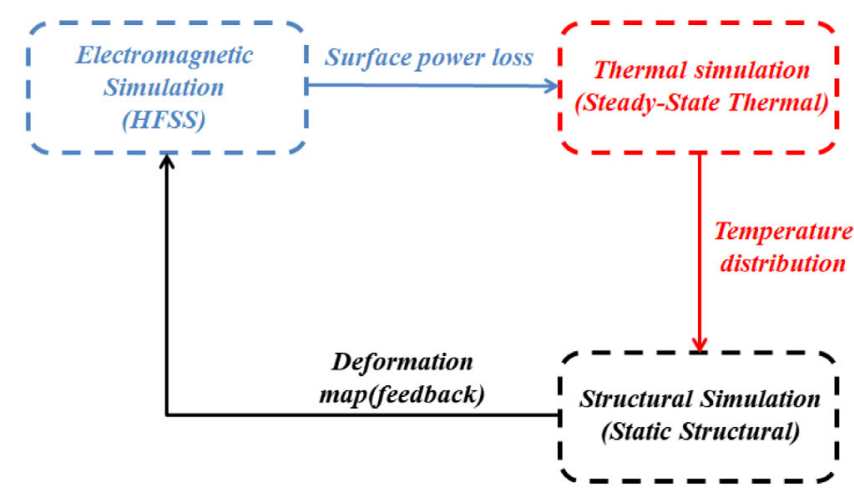

FIG. 21. The coupled multiphysics analyses procedure in the ANSYS Workbench.
TABLE VI. The power loss in different parts of the prototype cavity.

\begin{tabular}{lcc}
\hline \hline Part & Power loss $[\mathrm{W}]$ & $\%$ \\
\hline Vane & 6973 & 69.8 \\
Wall & 2757 & 27.6 \\
Tuner & 260 & 2.6 \\
\hline \hline
\end{tabular}

cooling channels were proposed for this prototype cavity, as shown in Fig. 22. All of the cooling channels are straight and rotational symmetry, which makes the manufacture procedure easier and inexpensive. Main thermodynamic parameters of the cooling water are summarized in Table VII. The diameter of the water channel is $10 \mathrm{~mm}$, the flow speed of the cooling water is about $2.3 \mathrm{~m} / \mathrm{s}$, the heat transfer coefficient is calculated by the empirical formulas [26], which is $9538 \mathrm{~W} /\left(\mathrm{m}^{2} \mathrm{~K}\right)$.

Just as what we had already done in the RF-EM design, multiphysics analyses of this BV-RFQ prototype cavity will begin with a slice model, and then extended to the full length model. Main concerns during the simulations were the temperature, deformation and stress distribution, and the frequency shift.

\section{A. Slice model analyses}

A $5 \mathrm{~mm}$ slice model with cooling channels was built for the multiphysics analyses, as shown in Fig. 23. The surface losses calculated in HFSS were scaled to the operation level (vane voltage equal to $70 \mathrm{kV}$ ) and transmitted to the steady-state thermal solver. Temperatures of the environment and the cooling water are set to $20^{\circ} \mathrm{C}$, the temperature distribution on the rf surface could be calculated and is shown in Fig. 24. The maximum temperature is

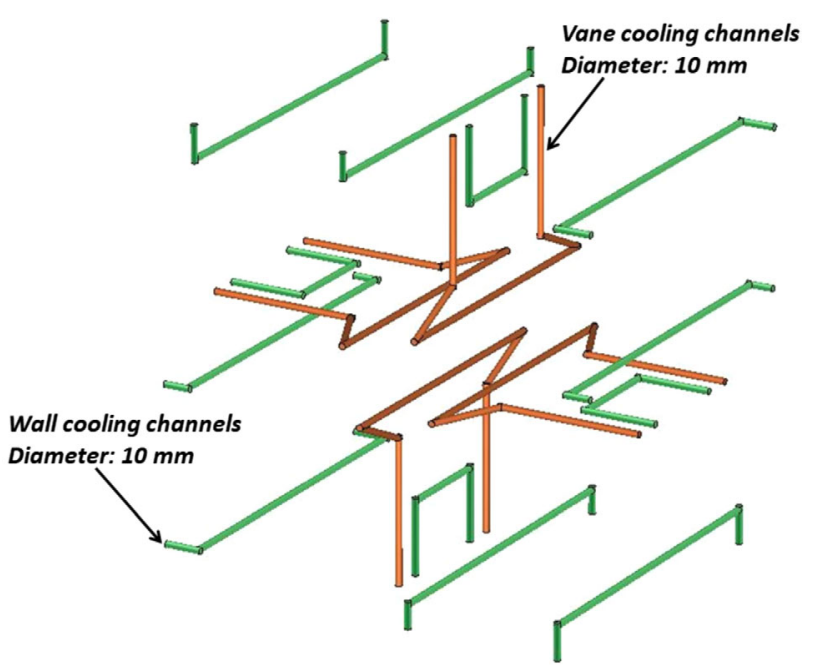

FIG. 22. The cooling channels distribution diagram of the vanes and walls in the prototype cavity. 
TABLE VII. The thermodynamic parameters of the cooling water in the vane and wall channels for the prototype cavity.

\begin{tabular}{lc}
\hline \hline Parameter & Value \\
\hline Diameter $[\mathrm{mm}]$ & 10 \\
Flow speed $[\mathrm{m} / \mathrm{s}]$ & 2.3 \\
Reynolds number & 23000 \\
Prandtl number & 6.644 \\
Nusselt number & 151.4 \\
Heat transfer coefficient $\left[\mathrm{W} /\left(\mathrm{m}^{2} \mathrm{~K}\right)\right]$ & 9538 \\
\hline \hline
\end{tabular}

$24.27^{\circ} \mathrm{C}$, which are located at the middle of the vanes. The minimum temperature is $22.245^{\circ} \mathrm{C}$, which are located at the cavity corners.

With this temperature map, the deformation and stress distributions were calculated in the static-structure solver and presented in Figs. 25 and 26. The maximum deformation is $35.426 \mu \mathrm{m}$, which are located at the cavity corners. The deformations at the regions near bent vane tips are ranging from 24 to $25.7 \mu \mathrm{m}$. The maximum stress is 8.455 $\mathrm{MPa}$, which are located in the middle of vanes, and is far below the yield strength of the copper. The deformation map is fed back to the HFSS and the frequency shift is calculated,which is $16.7 \mathrm{kHz}$ compared with the initial frequency.

Fixing the wall cooling water temperature to $20^{\circ} \mathrm{C}$, changing the vane cooling water temperature from $10^{\circ} \mathrm{C}$ to $30^{\circ} \mathrm{C}$, or altering this procedure, the frequency sensitivity for different cooling water temperatures were calculated, as shown in Fig. 27. It can be seen clearly that the frequency sensitivity is $-2.83 \mathrm{kHz} /{ }^{\circ} \mathrm{C}$ for the vane cooling water and $2.82 \mathrm{kHz} /{ }^{\circ} \mathrm{C}$ for the wall cooling water. The frequency shift of the prototype cavity can be adjusted with the changes of vane and wall cooling water temperature in operation.

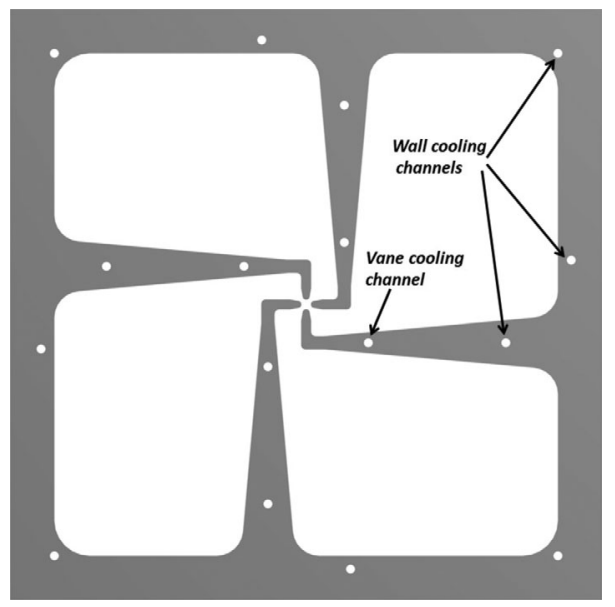

FIG. 23. The slice model with vane and wall channels.

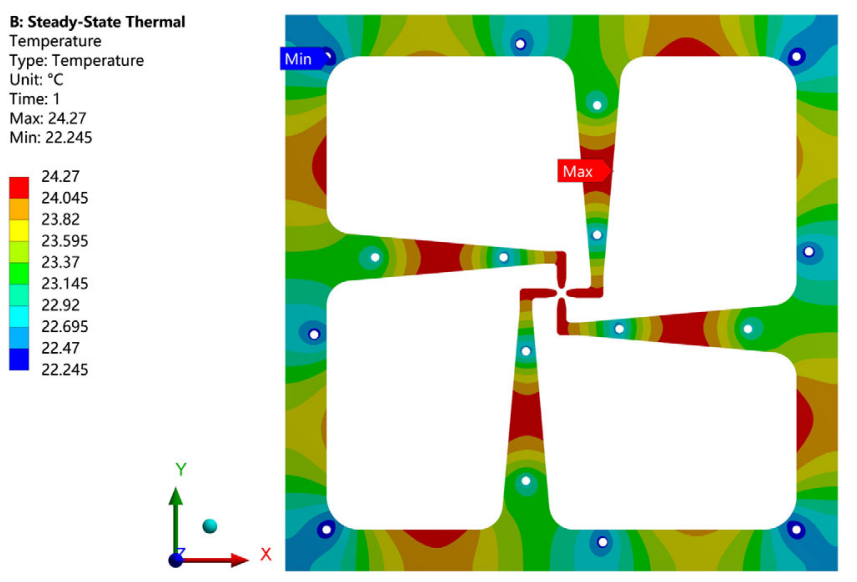

FIG. 24. The temperature distribution.
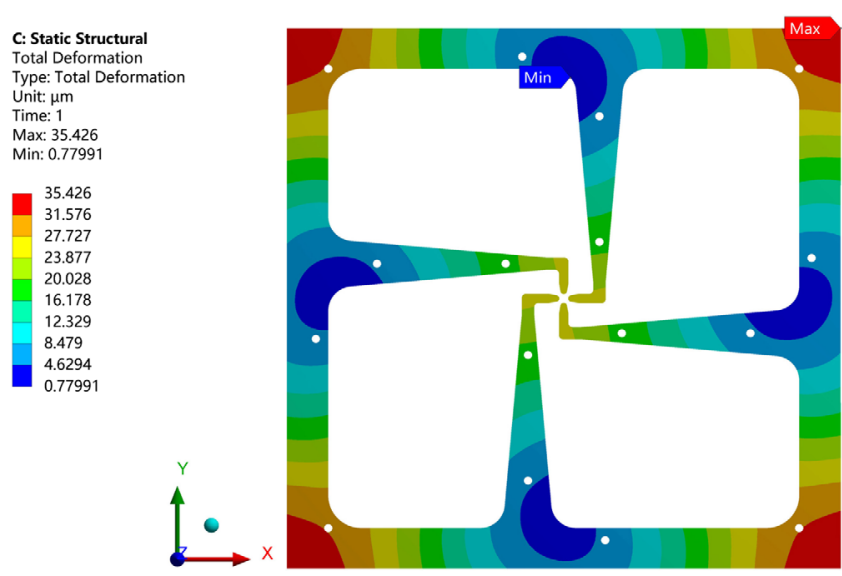

FIG. 25. The deformation map.

\section{B. Full length model analyses}

Further multi-physics analyses were done on a complete model which includes all eight tuners, four vane cooling channels, twelve wall cooling channels and two end plates, as shown in Fig. 28. The insertion depth of these tuners are

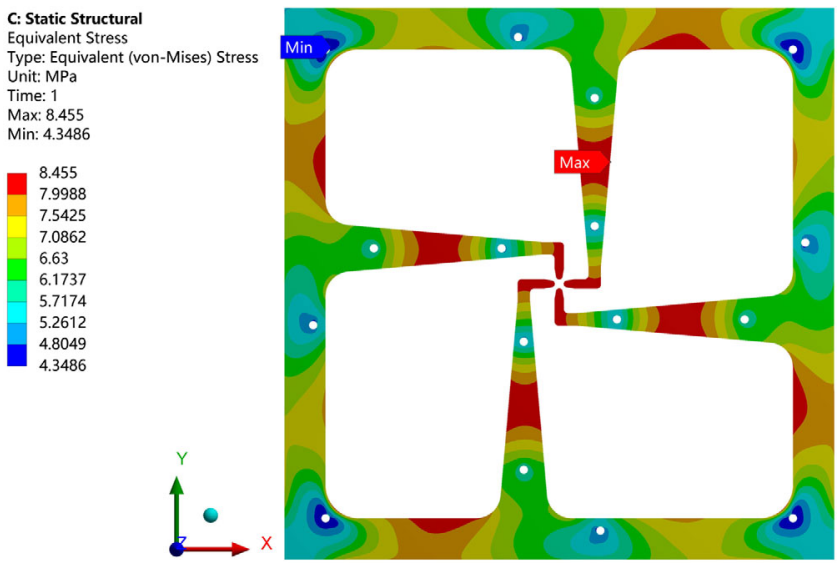

FIG. 26. The stress profile. 


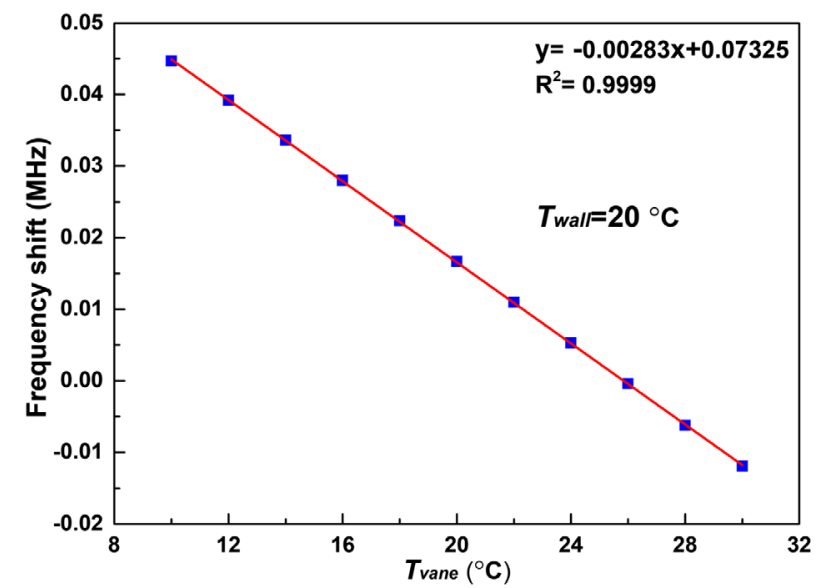

(a)

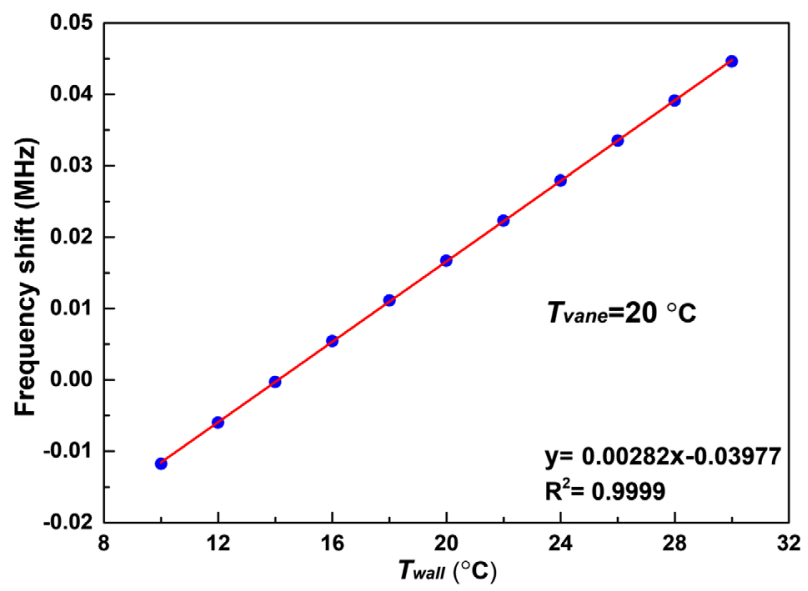

(b)

FIG. 27. The frequency shift for different cooling water temperature, (a) varying the vane cooling water temperature with $T_{\text {wall }}=20^{\circ} \mathrm{C}$; (b) varying the wall cooling water temperature with $T_{\text {vane }}=20^{\circ} \mathrm{C}$.

$25 \mathrm{~mm}$. The thickness of two end plates and the outer wall is $50 \mathrm{~mm}$, thus resulted to a complete model length equal to $650.07 \mathrm{~mm}$.

Figure 29 shows the temperature distribution of the complete model by the steady-state thermal solver. The maximum temperature is $33.917^{\circ} \mathrm{C}$, which are located at the undercuts. The minimum temperature is $22.402{ }^{\circ} \mathrm{C}$, which are located at the cavity corners.

Considering the real operation situation, along with the temperature distribution, atmospheric pressure on all outer surfaces and DOF (degree of freedom) constraint of fixed surface were added to perform the calculation during the deformation and stress analyses. The deformation and stress distributions are described in Figs. 30 and 31. With the DOF constraint of the fixed surface, deformations of the bent vane tips are asymmetrical. The deformations on

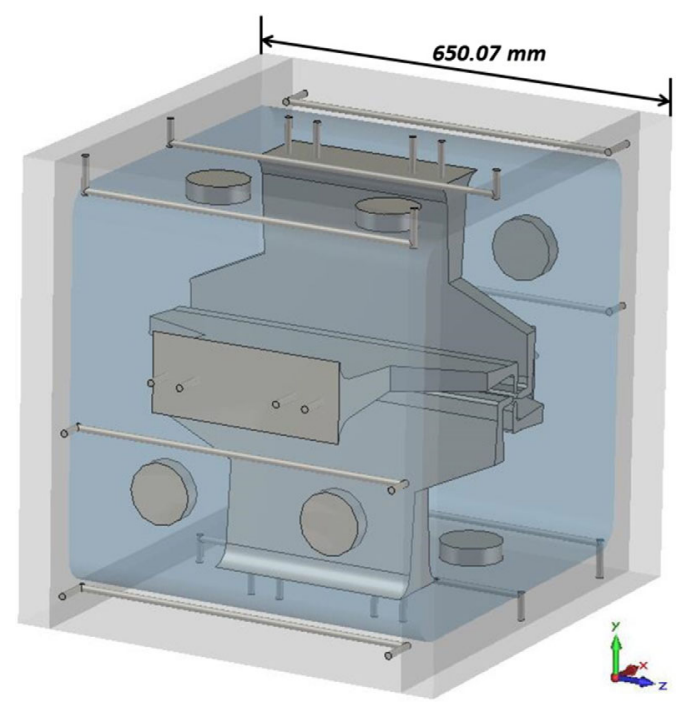

FIG. 28. The complete BV-RFQ model for the multiphysics analysis. the bent vane tips region are ranging from 30 to $60 \mu \mathrm{m}$. The maximum defamation is $78.216 \mu \mathrm{m}$, which is located at the end plate (entrance). The maximum stress is $14.682 \mathrm{MPa}$, which is located at the end plate (exit), and is far below the yield strength of the copper.

With the deformation distribution, the frequency shift of the complete model was calculated by HFSS. The frequency shift is $-16.6 \mathrm{kHz}$ compared with the initial frequency. Such a small frequency shift could be fully compensated by the tuners or the cooling water.

From the above discussions, the cooling system is proved to be very simple but effective to eliminate frequency shift due to thermal deformation.

\section{Tolerance study}

Ideal cavity models were adopted in the multiphysics analyses, and the vane voltage was scaled to $70 \mathrm{kV}$. The shunt impedance was calculated with perfect copper, thus resulted to a total power loss of $9990 \mathrm{~W}$. Tolerance

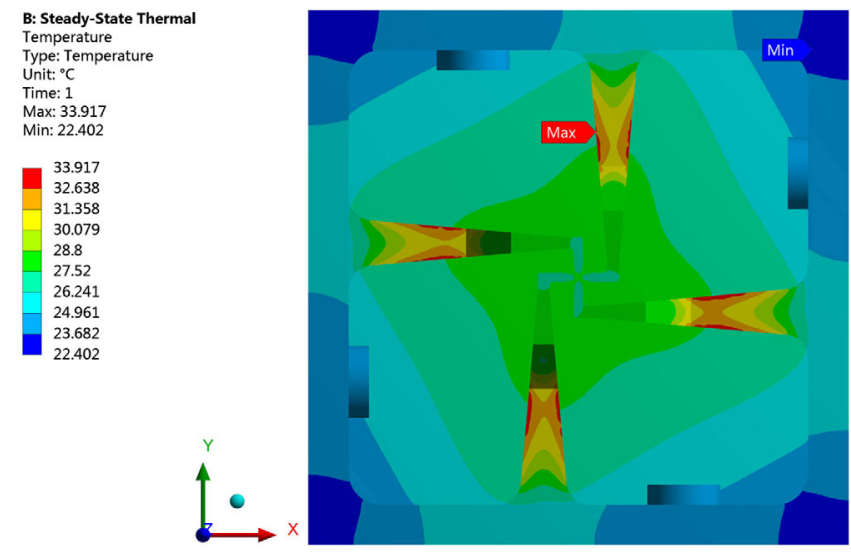

FIG. 29. The temperature distribution of the BV-RFQ prototype cavity. 


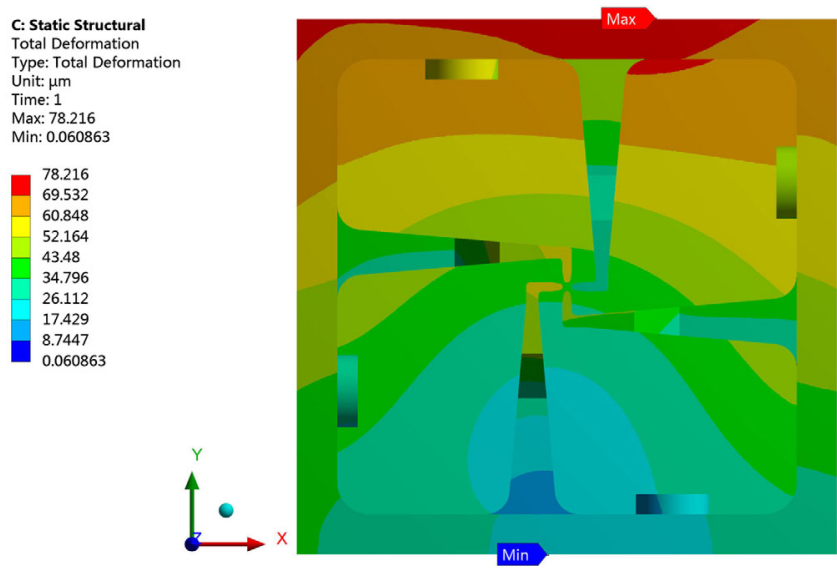

FIG. 30. The deformation distribution of the BV-FFQ prototype cavity.

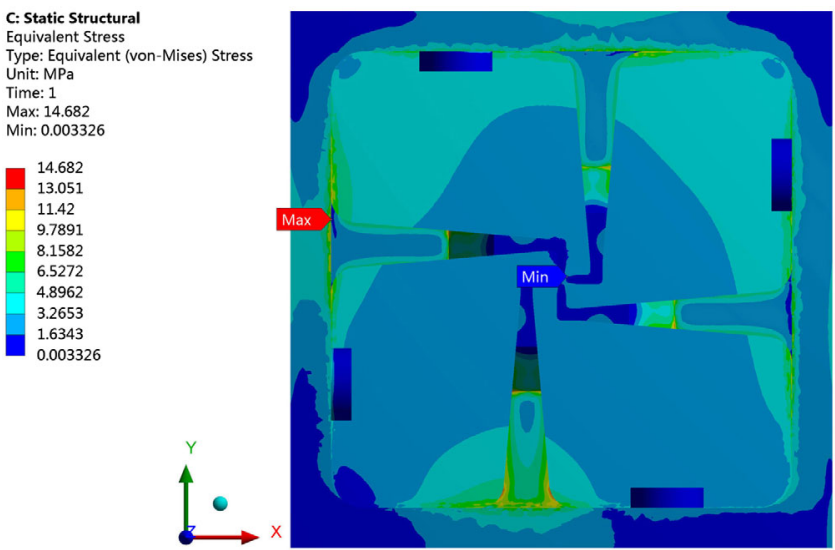

FIG. 31. The stress distribution of the BV-RFQ prototype cavity.

studies on the maximum temperature, maximum deformation, maximum stress, and frequency shift were done from $5 \%$ to $42.8 \%$ power loss increase, as shown in Fig. 32. From the simulation results, the temperature increase is about $6{ }^{\circ} \mathrm{C}$ from the design point to $42.8 \%$ power loss

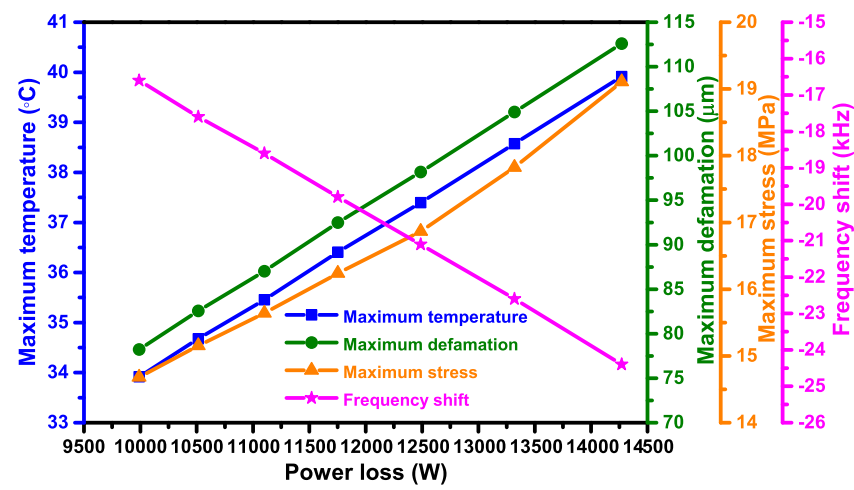

FIG. 32. The tolerance study of the multi-physics analyses of the BV-RFQ prototype cavity.

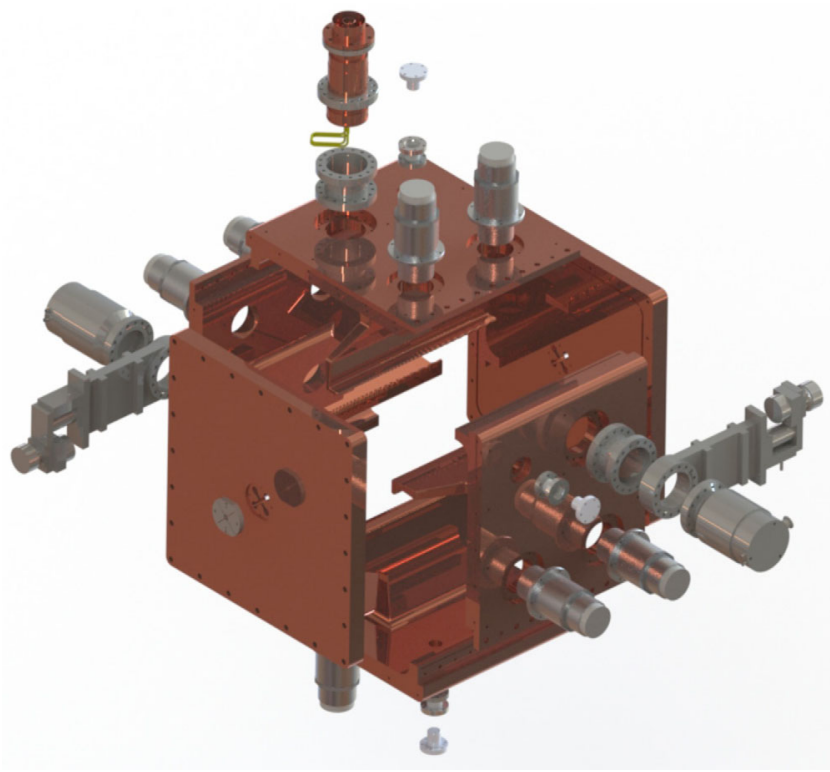

FIG. 33. The mechanical structure of the prototype cavity.

increase, which results to additional $34 \mu \mathrm{m}$ deformation and 4.5 MPa stress. Frequency shift increase due to the additional deformation is about $7.8 \mathrm{kHz}$, which could be easily tuned. Maximum stress is only $19 \mathrm{MPa}$ even at the worst case $(14271 \mathrm{~W})$, which is still far below the copper yield strength.

\section{MECHANICAL DESIGN OF THE PROTOTYPE}

The mechanical structure of this BV-RFQ prototype with cooling channels and variety ports is presented in Fig. 33. The cavity is divided into four modules during the assembling. Two vacuum ports, one fundamental power coupler port, two pickup antenna ports, eight tuner ports and four monitor ports are proposed on the outer wall of the cavity. A cold model prototype will be manufactured firstly to verify the manufacture and processing procedure, and the electromagnetic field distribution. An engineering model is planned in the next step.

\section{SUMMARY AND OUTLOOK}

A novel RFQ resonant structure with bent vanes was envisaged at IMP to get a compact cross section size at low frequency. An $81.25 \mathrm{MHz}$ proof-of-principle cavity was proposed to demonstrate this innovative concept.

The prototype cavity was proposed to accelerate $81.25 \mathrm{MHz} \mathrm{He}^{+}(q / A=1 / 4)$ bunches from $4 \mathrm{keV} / \mathrm{u}$ to $42.5 \mathrm{keV} / \mathrm{u}$ with vane length of $530.07 \mathrm{~mm}$. With careful beam dynamics optimizations, $96.9 \%$ transmission efficiency could be achieved for $5 \mathrm{~mA} \mathrm{He}{ }^{+}$beam. Tolerance analyses show that the beam dynamics design keeps great performance with a large margin.

The cross section size of the $81.25 \mathrm{MHz}$ BV-RFQ could be reduced by $40 \%$ with a 105 -degree bend angle compared 
with the normal four-vane type RFQ at the same frequency, along with a reduction of $47 \%$ on the quality factor. Considering the cavity size, quality factor dropping, and the machining difficulty, the bent angle was chosen to be 90 degrees finally, which resulted to a cross section size reduction of $20 \%$ and a quality factor dropping of $27 \%$.

The cross section of the vane profile of the BV-RFQ could be defined and optimized with 13 independent variables. Enough tuning range $(-276.97-483.12 \mathrm{kHz})$ was realized with eight optimized tuners, and a flat electric field distribution was achieved with appropriate undercuts design in the full length model. Mode separation is unnecessary for this prototype cavity since the nearest dipole mode is away from the operation quadrupole mode.

The beam dynamics design of this prototype cavity was verified in TraceWin with the electromagnetic field maps exported from CST MWS for the full length model. It was proved that the cavity is operated on the $T E_{210}$-like mode, thus the particles could be bunched and accelerated at the same time with suitable vane modulations.

Multipacting seems could be found in almost all power points. It is crucial for us to pay special attentions during the rf conditioning. Further optimizations on the rf structure to reduce the multipacting occurrence are on the way.

A simple cooling system was designed to meet requirements for the prototype cavity in $\mathrm{CW}$ operation. The temperature rise, deformation and stress could be controlled in secure levels. The frequency shift is relatively small and could be easily eliminated by the change of the cooling water temperature or by the adjustments of tuners. The sensitivity of the cavity frequency to the temperature of the cooling water on the vanes is almost the same to that on the walls but with opposite direction. Frequency stability of the prototype cavity could be kept by adjusting the temperatures of cooling water on vanes and walls during the commissioning.

The fabrication of the prototype cavity cold model is in progress. An engineering model is planned in the next step.

\section{ACKNOWLEDGMENTS}

The primary author, Lei Yang, would like to thank Dr. Wei Ma, Dr. Xiaowen Zhu, Dr. Shuhui Liu, and Dr. Haipeng Li for helpful discussions and suggestions on the beam dynamics simulation of the prototype cavity. This work is supported by the National Natural Science Foundation of China (Grants No. 11675236, No. 11475232, and No. 11535016).

[1] U. Bartz and A. Schempp, The new CW RFQ prototype, in Proceedings of the 25th International Linear Accelerator Conference, LINAC-2010, Tsukuba, Japan, 2010 (JACoW, Geneva, 2010), pp. 488-490.
[2] D. Koser, P. Gerhard, and H. Podlech, Mechanical vibration analysis of the 4-rod RFQ at the high charge state injector at GSI, Nucl. Instrum. Methods Phys. Res., Sect. A 917, 47 (2019).

[3] H. Podlech, K. Kümpel, C. Lorey et al., The MYRRHARFQ - status and first measurements, in Proceedings of the 8th International Particle Accelerator Conference, IPAC2017, Copenhagen, Denmark, 2017 (JACoW, Geneva, 2017), pp. 2244-2245.

[4] N. Bultman, E. Pozdeyev, G. Morgan et al., Design of the FRIB RFQ, in Proceedings of the 4th International Particle Accelerator Conference, IPAC-2013, Shanghai, China, 2013 (JACoW, Geneva, 2013), pp. 2866-2868.

[5] W. Ma, L. Lu, X. B. Xu, L. Sun, Z. Zhang, W. Dou, C. Li, L. Shi, Y. He, and H. Zhao, Design of an $81.25 \mathrm{MHz}$ continuous-wave radio-frequency quadrupole accelerator for Low Energy Accelerator Facility, Nucl. Instrum. Methods Phys. Res., Sect. A 847, 130 (2017).

[6] W. Ma, L. Lu, T. Liu, X. Xu, L. Sun, C. Li, L. Shi, W. Wang, Y. He, and H. Zhao, Three-dimensional multiphysics analysis and commissioning frequency tuning strategy of a radio-frequency quadrupole accelerator, Nucl. Instrum. Methods Phys. Res., Sect. A 866, 190 (2017).

[7] W. Ma, L. Lu, T. Liu et al., Frequency and fields tuning of a heavy ion radio-frequency quadrupole accelerator, Nucl. Instrum. Methods Phys. Res., Sect. A 901, 180 (2018).

[8] P. N. Ostroumov and A. A. Kolomiets, Design of $57.5 \mathrm{MHz}$ cw RFQ for medium energy heavy ion superconducting linac, Phys. Rev. Accel. Beams 5, 060101 (2002).

[9] T. P. Wangler, $R F$ Linear Accelerators (Wiley-VCH, Berlin, 2008).

[10] K. R. Crandall, T. P. Wangler, L. M. Young et al., RFQ Design Codes, the RFQGen is a beam-dynamics code for the RFQ linear accelerator, and it is developed by Los Alamos National Laboratory, 2005.

[11] CST, http://www.cst.com.

[12] L. Yang, L. Lu, Z. L. Zhang et al., Research on the new cavity structure of RFQ accelerator with bent vanes at IMP, in Proceedings of the 29th International Linear Accelerator Conference, LINAC-2018, Beijing, China, 2018 (JACoW, Geneva, 2018), pp. 802-804.

[13] P. P. Gan, K. Zhu, Q. Fu et al., Development and beam commissioning of a continuous-wave window-type radio-frequency quadrupole, Phys. Rev. Accel. Beams 22, 030102 (2019).

[14] A. Plastun, V. Andreev, V. Koshelev et al., RF design of the NUCLOTRON-NICA 145.2 MHz RFQ, in Proceedings of the 28th International Linear Accelerator Conference, LINAC-2016, East Lansing, MI, USA, 2016 (JACoW, Geneva, 2016), pp. 596.

[15] S. Abbott, D. Brodzik, R. A. Gough et al., RFQ development at LBL, IEEE Trans. Nucl. Sci. 30, c2 (1983).

[16] A. Schempp, H. Deitinghoff, H. Klein et al., The CRYRING RFQ, in Proceedings of the 14th International Linear Accelerator Conference, LINAC-1988, Williamsburg, Virginia, USA, 1988 (JACoW, Geneva, 1988), pp. 70-72.

[17] M. Pasini, O. Kester, D. Habs et al., RF design of the MAFF IH-RFQ, in Proceedings of the 9th European Particle Accelerator Conference, EPAC-2004, Lucerne, Switzerland, 2004 (JACoW, Geneva, 2004), pp. 1216-1217. 
[18] C. M. Kleffner, M. Grieser, D. Habs et al., The RFQAccelerator for the High Current Injector of the TSR, in Proceedings of the 3th European Particle Accelerator Conference, EPAC-1992, Berlin, Germany, 1992 (JACoW, Geneva, 1992), pp. 1340-1342.

[19] http://rex-isolde.web.cern.ch/rfq.

[20] P. Fischer and A. Schempp, Tuning a CW 4-rod RFQ, in Proceedings of the 23th International Linear Accelerator Conference, LINAC-2006, Knoxville, Tennessee USA, 2006 (JACoW, Geneva, 2006), pp. 728.

[21] U. Bessler, A. Schempp, T. Sieber et al., Experiments with heavy-ion beams and RF-tests with the $27 \mathrm{MHz}$ high-current spiral-RFQ-prototype, in Proceedings of the 18th International Linear Accelerator Conference, LINAC-
1996, Geneva, Switzerland, 1996 (JACoW, Geneva, 1996), pp. 56-58.

[22] D. Uriot and N. Pichoff, TraceWin documentation, CEA/ SACLAY/DSM/Irfu/SACM, 2015.

[23] M. A. Furman and M. T. F. Pivi, Probabilistic model for the simulation of secondary electron emission, Phys. Rev. Accel. Beams 5, 124404 (2002).

[24] X. W. Zhu, H. Wang, Y. R. Lu, Z. Wang, K. Zhu, Y. Zou, and Z. Guo, 2.5 MeV CW 4-vane RFQ accelerator design for BNCT applications, Nucl. Instrum. Methods Phys. Res., Sect. A 883, 57 (2018).

[25] ANSYS, http://www.ansys.com.

[26] F. Kreith, The CRC Handbook of Thermal Engineering (CRC Press, Boca Raton, 2000). 